\title{
Symmetries of Einstein-Yang-Mills Fields and Dimensional Reduction
}

\author{
R. Coquereaux and A. Jadczyk \\ Centre de Physique Théorique ${ }^{\star}$, Section II, CNRS - Luminy - Case 907, F-13288 Marseille, \\ Cedex 9, France
}

\begin{abstract}
Let $E$ be a manifold on which a compact Lie group $S$ acts simply (all orbits of the same type); $E$ can be written locally as $M \times S / I, M$ being the manifold of orbits (space-time) and $I$ a typical isotropy group for the $S$ action. We study the geometrical structure given by an $S$-invariant metric and an $S$-invariant Yang Mills field on $E$ with gauge group $R$. We show that there is a one to one correspondence between such structures and quadruplets $\left(\gamma_{\mu \nu}, A_{\mu}^{\hat{\alpha}}, \phi_{\alpha}^{i}, h_{\alpha \beta}\right)$ of fields defined solely on $M ; \gamma_{\mu \nu}$ is a metric on $M, h_{\alpha \beta}$ are scalar fields characterizing the geometry of the orbits (internal spaces), $\phi_{\alpha}^{i}$ are other scalar fields (Higgs fields) characterizing the $S$ invariance of the $\operatorname{Lie}(R)$-valued Yang Mills field and $A_{\mu}^{\hat{\alpha}}$ is a Yang Mills field for the gauge group $N(I) \mid I \times Z(\lambda(I)), N(I)$ being the normalizer of $I$ in $S, \lambda$ is a homomorphism of $I$ into $R$ associated to the $S$ action, and $Z(\lambda(I))$ is the centralizer of $\lambda(I)$ in $R$. We express the Einstein-Yang-Mills Lagrangian of $E$ in terms of the component fields on $M$. Examples and model building recipes are given.
\end{abstract}

\section{Introduction}

\section{I.1. Several Descriptions for the Same Geometrical Structure}

Symmetry properties of gravity (metric structure) and Yang-Mills fields (connections) have been often studied separately, both by physicists and mathematicians. These two kinds of geometrical structures are however deeply inter-related and several techniques of "dimensional reduction" allow us to cast a new light on the subject. Let us suppose that we live in an extended universe $U$ endowed with a metric $g(U)$ invariant under a group $G$ (description 1), then, in many cases, we can also describe the same situation by saying that we live in an universe $E$ $(\operatorname{dim} E<\operatorname{dim} U)$ endowed with a metric $g(E)$ and a Yang-Mills field $A(E)$, both invariant under a subgroup of $G$ (description 2). We can finally describe the same

* Institute of Theoretical Physics, University of Wroclaw (Poland)

$\star \star$ Laboratoire Propre, Centre National de la Recherche Scientifique 
situation by saying that we live in a universe $M$ (space-time, $\operatorname{dim} M<\operatorname{dim} E<\operatorname{dim} U)$ endowed with a metric $g(M)$, a new Yang-Mills field $A(M)$, a few scalar fields and no symmetries left (description 3$)$. The study of the link between descriptions 2 and 3 is the aim of this paper. The method that we shall use is the following: a general result [1], which we recall in Sect. 2, allows us to obtain the link between descriptions 1 and 2 as well as the link between descriptions 1 and 3, we will therefore obtain the desired results by comparing the above two relations. Particular examples of the general situation have been studied in $[2,3]$ providing interesting phenomenological models; the interpretation of Higgs fields as Yang-Mills fields has been emphasized in [4-7] where some properties of symmetric Yang-Mills fields are also studied. The construction given in the present paper is a natural application of the methods developed in [1] and may be thought of as an alternative to that of [8], where a direct analyzis of the link between the descriptions 2 and 3 is made (see also [9]).

\section{I.2. The Mathematical Framework}

Symmetries can be studied globally (group actions) or locally (vector fields); here we study symmetric configurations of coupled Yang-Mills and Einstein fields in the most general case and we perform this analysis both from the local and global point of view. The natural mathematical framework used to study global aspects is provided by the theory of fiber bundles and we will use freely the corresponding terminology. In plain terms, let us only say that a fiber bundle is a geometrical object which can be thought of as a collection of "fibers" glued together and parametrized by a manifold called the "basis"; besides, often there is a well defined action of a group $G$ on the fibers (the "structural group")-one can think of the base as being space-time and of the typical fiber as being the internal space (there is an internal space above every space-time point). Properties of connections are discussed in the mathematical literature in terms of the connection form (which is a Lie algebra valued one-form on the bundle space) but physicists prefer to use Yang-Mills fields (which are Lie algebra valued one-forms on the basis); YangMills fields can only be defined (locally) via the choice of a (local) gauge ("section" of the bundle). In the following we will express the results in these two languages.

\section{I.3. Structure of the Paper}

In Sect. 2, we show how to construct invariant metrics on fiber bundles and recall the Reduction Theorem [1]. In Sect. 3, we define and study symmetries of bundles and connections. In Sect. 4, we analyze the geometrical structure for a space $E$ endowed with an $S$-invariant metric and a $S$-invariant, $\operatorname{Lie}(R)$-valued Yang-Mills field; we obtain a generalized reduction theorem and a "dimensionally reduced" Einstein-Yang-Mills action. In Sect. 5, we discuss examples, model building recipes and give comments and a summary of our results. The reader who only wants to get the main ideas of the present paper may read only the summary section (5.1) as well as Tables 1, 2 (Sect. 4.2). Whenever we give a physical interpretation to our results, the signature of space-time is +++- , see also Sect. 5.4. 


\section{Invariant Metrics on Fiber Bundles}

\subsection{Invariant Metrics on Principal Bundles}

Let us first recall a result which is well known both in physical and in the mathematical folklore (see e.g. [10-12]; consider a $G$-invariant metric $g(P)$ on a principal bundle $P$ of base $M$ and structure group $G$, then this metric can be reinterpreted in terms of objects defined on $M$ : a metric $g(M)=\left(\gamma_{\mu \nu}\right)$ on $M$, a gauge field $A(M)=\left(A_{\mu}^{\alpha}(x)\right), \alpha=1,2, \ldots, n=\operatorname{dim} \mathscr{G}$, valued in $\mathscr{G}=\operatorname{Lie}(G)$, and $\frac{n(n+1)}{2}-$ component scalar field $h \equiv\left(h_{\alpha \beta}(x)\right)$ which, for a fixed $x$, determines a rightinvariant metric in the copy of $G$ above $x$. Reciprocally, the data consisting of these three objects allow us to reconstruct back a unique $G$-invariant metric on the bundle. For example, if we believe that the "real world" can be described by a space which is a "local product" of space-time $M$ and the color group SU(3) - the internal space - then an SU(3)-invariant metric on this $4+8=12$-dimensional space $P$ splits into a gravity field on $M$, a color field, and scalar fields $h_{\alpha \beta}(x)$ characterizing the "shape" of the internal SU(3) space above $x \in M[$ notice that there is a $\frac{8 \times 9}{2}=36$-parameter family of right invariant metrics on $\left.\mathrm{SU}(3)\right]$.

The scalar curvature $\tau_{P}$ associated to a $G$-invariant metric on $P$ is constant along the fibers and can be written entirely in terms of the fields on $M^{1}$

$$
\begin{aligned}
\tau_{x}(P)= & \tau_{x}(M)+\tau_{x}(G)-\frac{1}{4} h_{\alpha \beta} F_{\mu \nu}^{\alpha} F^{\mu \nu \beta} \\
& -\frac{1}{4} h^{\alpha \beta} h^{\gamma \delta}\left(D_{\mu} h_{\alpha \gamma} D^{\mu} h_{\beta \delta}+D_{\mu} h_{\alpha \beta} D^{\mu} h_{\gamma \delta}\right) \\
& -\nabla_{\mu}\left(h^{\alpha \beta} D^{\mu} h_{\alpha \beta}\right), \\
D_{\mu} h_{\alpha \beta}= & \partial_{\mu} h_{\alpha \beta}+C_{\alpha \gamma}^{\delta} A_{\mu}^{\gamma} h_{\delta \beta}+C_{\beta \gamma}^{\delta} A_{\mu}^{\gamma} h_{\alpha \delta},
\end{aligned}
$$

where $C_{\alpha \beta}^{\gamma}$ are the structure constants for $G$.

$\tau_{x}(M)$ is the scalar curvature of $M$, and $\tau_{x}(G)$ is the scalar curvature of the copy of $G$ over $x$, which can be interpreted as (minus) the potential term for the scalar fields ${ }^{2}$

$$
\tau_{G}(x)=-h^{\alpha \alpha^{\prime}}\left(\frac{1}{2} C_{\alpha \beta}^{\gamma} C_{\alpha^{\prime} \gamma}^{\beta}+\frac{1}{4} h^{\beta \beta^{\prime}} h_{\gamma \gamma^{\prime}} C_{\alpha \beta}^{\gamma} C_{\alpha^{\prime} \beta^{\prime}}^{\gamma^{\prime}}+C_{\alpha \beta}^{\beta} C_{\alpha^{\prime} \gamma}^{\gamma}\right) .
$$

If one assumes that $G$ [SU(3) in our example] takes, at each point $x \in M$, its most symmetric standard shape (the $G \times G$-invariant Killing metric), then $D_{\mu} h_{\alpha \beta} \equiv 0$, and we recover the usual Einstein-Yang-Mills action; in that case $\tau_{G}$ is just a (cosmological) constant.

Let us end this subparagraph with a mathematical application: construction of all possible SU(2)-invariant metrics on the seven sphere $S^{7}$. First we realize that $S^{7}$ can be written as an SU(2) bundle over $S^{4}$ (the Hopf fibration), therefore a direct use of the above theorem tells us that in order to construct a general SU(2)-

1 The factor in the kinetic energy term of scalars is $-\frac{1}{4}$ and not $-\frac{1}{2}$ as erroneously printed in [1]

2 The last term in (2.1.2) vanishes for all unimodular groups and will be omitted in the rest of this paper 
invariant metric on $S^{7}$ one must choose 1) an arbitrary metric on $S^{4}, 2$ ) an arbitrary Yang-Mills field defined on $S^{4}$ with values in Lie (SU(2)), and 3) an arbitrary $\mathrm{SU}(2)$-invariant metric on each copy of $\mathrm{SU}(2)$ above the points of $S^{4}$ (there is a 6-parameter family of such metrics).

Remark 2.1.1. The last term of the Eq. (2.1.1) gives no contribution to the field equation if one takes for the Lagrangian $L \approx \tau_{P} \sqrt{|g(M)|}$; however, it has to be taken into account if the volume density is taken to be $\sqrt{|g(P)|}$.

\subsection{Invariant Metrics on Bundles with Homogeneous Fibers}

What we will need in the following is actually a generalization of the previous theorem to the case when the internal space is not a group $G$ but, more generally, a homogeneous space $\mathrm{G} / \mathrm{H}{ }^{3}$ Such a generalization was obtained in [1], where the following result (which we call the Reduction Theorem) was proved.

Reduction Theorem. Consider a right action of $G$ on a local product bundle $E \simeq M \times(G / H)$ with base $M$, whose fibers are homogeneous spaces isomorphic to $G / H$. Then there is a one-to-one correspondence between $G$-invariant metrics on $E$ and triples $\left(\gamma_{\mu \nu}, A_{\mu}^{\hat{\alpha}}, h_{\alpha \beta}\right)$, where $\gamma_{\mu \nu}$ is a metric on the base $M, A_{\mu}^{\hat{\alpha}}$ is a Yang-Mills potential with gauge group $N(H) \mid H, N(H)$ being the normalizer of $H$ in $G$, and the scalar fields $h_{\alpha \beta}(x)$ describe a $G$-invariant metric in the copy of $G / H$ above $x$.

Let us recall that the normalizer $N(H)$ of $H$ in $G$ is the biggest subgroup of $G$ in which $H$ is invariant; we have $H=\{a \in G: a H=H a\}$. The tangent space at the origin of $G / H$ (indices $\alpha, \beta, \ldots$ ) is isomorphic to $\mathscr{G} / \mathscr{H}$ and is usually not a Lie algebra, but it contains as a subspace the Lie algebra of $N(H) / H$ (we use $\hat{\alpha}, \hat{\beta}, \ldots$ for the indices of this subspace). The scalar curvature of $E$ endowed with such a $G$-invariant metric, can be, here again, expressed entirely in terms of $M$-based fields, and we get [1] an expression similar to Eq. (2.1.1)

$$
\begin{aligned}
\tau(E)= & \tau_{x}(M)+\tau_{x}(G / H)-\frac{1}{4} h_{\hat{\alpha} \hat{\beta}} F_{\mu \nu}^{2} F^{\mu \nu \hat{\beta}} \\
& -\frac{1}{4} h^{\alpha \beta} h^{\gamma \delta}\left(D_{\mu} h_{\alpha \gamma} D^{\mu} h_{\beta \delta}+D_{\mu} h_{\alpha \beta} D^{\mu} h_{\gamma \delta}\right)-\nabla_{\mu}\left(h^{\alpha \beta} D^{\mu} h_{\alpha \beta}\right),
\end{aligned}
$$

where

$$
D_{\mu} h_{\alpha \beta}=\partial_{\mu} h_{\alpha \beta}+C_{\alpha \hat{\gamma}}^{\delta} A_{\mu}^{\hat{\gamma}} h_{\delta \beta}+C_{\beta \hat{\gamma}}^{\delta} A_{\mu}^{\hat{\gamma}} h_{\alpha \delta}
$$

and

$$
\tau_{G / H}=-h^{\alpha \alpha^{\prime}}\left(\frac{1}{2} C_{\alpha \beta}^{\gamma} C_{\alpha^{\prime} \gamma}^{\beta}+\frac{1}{4} h^{\beta \beta^{\prime}} h_{\gamma \gamma^{\prime}} C_{\alpha \beta}^{\gamma} C_{\alpha^{\prime} \beta^{\prime}}^{\gamma^{\prime}}+C_{\alpha \beta}^{\gamma} C_{\alpha^{\prime} \underline{\gamma}}^{\beta}\right) .
$$

The index $\gamma$ in (2.2.2) runs through the Lie subalgebra $\mathscr{H} \subset \mathscr{G}$ of the stability group: $\mathscr{H}=\operatorname{Lie}(H)$.

Example 1. If we take as a model a space $E$ which is a local product of space-time $M$ and the 16-dimensional complex Stiefel manifold SU(5)/SU(3), then an SU(5)invariant metric on this 20-dimensional space $E$ can be constructed out of a gravity field on $M$, a Yang-Mills field with gauge group $\mathrm{SU}(2) \times \mathrm{U}(1)$, and a scalar field

3 Throughout this paper $G / H$ denotes the space of right cosets $\{H a: a \in G\}$. This is opposite to the usual convention 
$h_{\alpha \beta}(x)$ characterizing the shape of the internal $\mathrm{SU}(5) / \mathrm{SU}(3)$ homogeneous space sitting above $x \in M$. Notice that the Lie algebra of the normalizer of $\mathrm{SU}(3)$ in $\mathrm{SU}(5)$ is indeed the Lie algebra of $\mathrm{SU}(3) \times \mathrm{SU}(2) \times \mathrm{U}(1)$.

Example 2. We take as a model the 11-dimensional space $E=\frac{S^{7} \times S^{7}}{\mathrm{SU}(2)}$, which is constructed as follows: by using the right action $p \in S^{7}, g \in \mathrm{SU}(2) p g \in S^{7}$ of $\mathrm{SU}(2)$ on $S^{7}$, we define the following diagonal right action of $\mathrm{SU}(2)$ on $S^{7} \times S^{7}:\left(p, p^{\prime}\right) \in S^{7} \times S^{7}, g \in \mathrm{SU}(2)\left(p g, p^{\prime} g\right) \in S^{7} \times S^{7}$, and define $E$ as the coset space obtained via the diagonal action; of course we use the fact that $S^{7}$ is indeed a SU(2) bundle over $S^{4}$ (Hopf fibration). Let us now show that $E$ can also be written as a non-trivial $S^{7}$ bundle over $S^{4}$; indeed if we define a left action of SU(2) on $S^{7}$ by $g p=p g^{-1}$ we can construct an associated bundle $\left\{\right.$ class $\left.\left(p, p^{\prime}\right) \mid p, p^{\prime} \in S^{7}\right\}$, where

$$
\operatorname{class}\left(p, p^{\prime}\right)=\left\{\left(p g, g^{-1} p^{\prime}\right) \mid g \in \mathrm{SU}(2)\right\}=\left\{\left(p g, p^{\prime} g\right) \mid g \in \mathrm{SU}(2)\right\} .
$$

This associated bundle therefore coincides with $E$ but now it has base $S^{4}$ and typical fiber $S^{7}$. Now the group $G=\operatorname{Sp}(2)$ acts transitively on $S^{7}$ $\left(S^{7}=G / H=\mathrm{Sp}(2) / \mathrm{Sp}(1)\right)$, the normalizer of $\mathrm{Sp}(1)$ in $\mathrm{Sp}(2)$ being $\mathrm{Sp}(1) \times \mathrm{SU}(2)$; then $G=\operatorname{Sp}(2)$ acts also on $E$, there is only one stratum, all the orbits are of the same type $\left(S^{7}\right)$ and the manifold of orbits is $S^{4}$. The most general $S p(2)$ invariant metric on $E$ can be constructed out of a gravity field (metric) on $S^{4}$, a Yang Mills field $A_{\mu}^{\hat{\alpha}}$ defined on $S^{4}$, valued in SU(2) and a scalar field $h_{\alpha \beta}(x)$ characterizing the $\mathrm{Sp}(2)$-invariant metric of the copy of $S^{7}$ above $x \in S^{4}$ (there is a 7-dimensional manifold of such metrics [1]). This example could be generalized to the study of $G$-invariant metrics on spaces of the type $E=\frac{G / H \times G / H}{N(H) \mid H}$ considered as bundles
over $G / N$ with typical fiber $G / H$.

Other examples are given in [1]. Notice that when $G / H$ is an isotropy irreducible space [i.e. when $\operatorname{Ad}(H)$ acts on $\mathscr{G} / \mathscr{H}$ real irreducibly], then $N(H) / H$ is discrete. In such a case the Yang-Mills potential is trivial. More material on the subject can be found in $[13,14]$.

\section{Symmetries of a Principal Bundle}

In this section we will introduce the principal concepts and notation used throughout the rest of the paper.

\subsection{Symmetries of Yang-Mills Fields}

Let $(U, \pi, E, R)$ be a principal bundle with base $E$, projection $\pi$, and Lie structure group $R$ acting on $U$ from the right. Let $S$ be a Lie group acting on $U$ from the left by bundle automorphisms, i.e.

$$
s(u r)=(s u) r, \quad \forall s \in S, \quad r \in R,
$$

see Fig. 1. We shall assume that the action of $S$ on $U$ is effective, i.e. $s u=u, \forall u \in U$ implies $s=e$. The action of $S$ on $U$ induces an action of $S$ on $E: s \pi(u) \equiv \pi(s u)$. This induced action on $E$ need not be effective - thus we allow for pure gauge transformations also called vertical automorphisms (cf. [15-17]). 
We will also use the right action of $S$ on $U$ and on $E$ defined by

$$
\begin{array}{ll}
u s \equiv s^{-1} u, & u \in U, \quad s \in S, \\
y s \equiv s^{-1} y, & y \in E, \quad s \in S .
\end{array}
$$

When a possibility of confusion can arise, we shall write $L_{s}$ and $R_{s}$ for the left and right action respectively.

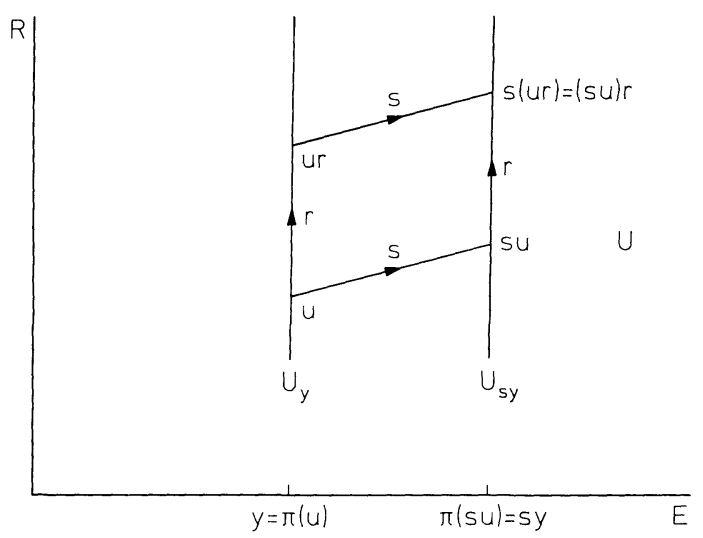

Fig. 1

Denote by $\mathscr{R}$ the Lie algebra of $R$, and let, for each $v \in \mathscr{R}, Z_{v}$ denote the fundamental vector field on $U$ generated by $v$ :

$$
Z_{v}(u)=\frac{d}{d t}[u \exp (t v)]_{t=0}
$$

Recall that a 1 -form $\omega$, defined on $U$ and with values in $\mathscr{R}$, is a 1 -form of a principal connection if $\omega\left(Z_{v}\right)=v$, and $R_{r}^{*} \omega=\operatorname{Ad}(r)^{-1} \omega, r \in R$. The group $S$, introduced above, is said to be a symmetry group of $\omega$ if, for all $s \in S$,

$$
R_{s}^{*} \omega=\omega
$$

The content of the above equation was discussed in many papers and we refer the reader to the existing literature $[6,7,18-21]$. The brief discussion we give below has the purpose of introducing notation and concepts we will use later on.

Global description of a gauge field involves a principal connection 1-form $\omega$. Locally a gauge field is described by an $\mathscr{R}$-valued 1 -form on $E$ rather than on $U$. Let $\sigma: E \rightarrow U$ be a local cross-section (gauge), then $A^{\sigma}$ - the Yang-Mills potential in the gauge $\sigma-$ is defined by $A^{\sigma} \equiv \sigma^{*} \omega$.

Let $S$ be a symmetry group of $\omega$, and let us see what can be said about the local representative $A^{\sigma}$. Of course the cross-section $\sigma$ will not be, in general, invariant under $S$. Its noninvariance is described by a compensating function $r \equiv r^{\sigma}(s ; y)$ 
taking values in the gauge group $R$, defined by (see Fig. 2):

$$
\sigma(y s)=[\sigma(y) s] r
$$

Fig. 2

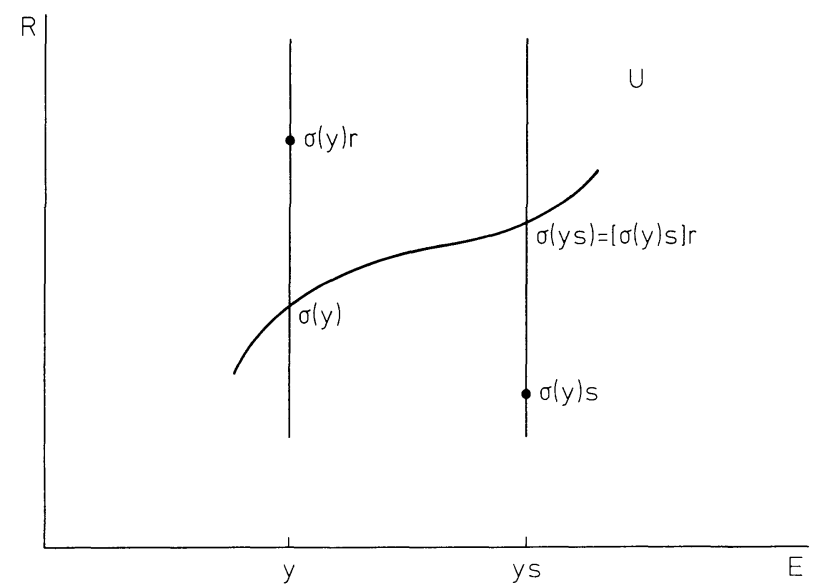

Because of noninvariance of the gauge $\sigma$, also the Yang-Mills potential $A^{\sigma}$ will not be invariant; indeed, using (3.1.1) we find

$$
R_{s}^{*} A^{\sigma}=\left(R_{s}^{-1} \circ \sigma \circ R_{s}\right)^{*} \omega=(\sigma r)^{*} \omega=A^{\sigma r}=r^{-1} A^{\sigma} r+r^{-1} d r .
$$

Therefore $R_{s}^{*} A^{\sigma}$ differs from $A^{\sigma}$ by a gauge transformation.

Let $\mathscr{S}$ be the Lie algebra of the symmetry group $S$. With $\xi \in \mathscr{S}$ let $t \mapsto \exp (t \xi)$ be the 1-parameter subgroup generated by $\xi$. Denote by $Z_{\xi}$ the fundamental vector field generated by $\xi$ :

$$
Z_{\xi}(y) \equiv \frac{d}{d t}[y \exp (t \xi)]_{t=0} .
$$

With $s=s(t)$ differentiate (3.1.3) with respect to $t$ at $t=0$. From the very definitions of Lie and covariant derivatives, we obtain $L_{z \xi} A^{\sigma}=D \Lambda^{\sigma}(\xi)$, where

$$
\Lambda^{\sigma}(\xi ; y) \equiv \frac{d}{d t}\left[r^{\sigma}(\exp (t \xi) ; y)\right]_{t=0} .
$$

For a fixed $y \in E, \Lambda^{\sigma}(\cdot ; y)$ is a linear map from $\mathscr{S}$ to $\mathscr{R}$. It will play an important role later on. Let us analyze it a little bit closer. Choose $y \in E$ and denote by $I$ the stability group of $y, I=\{s \in S: s y=y\}$. Choose $u \in \pi^{-1}(y)$, and let, for every $s \in I$, $\lambda_{u}(s)$ be the unique element of $R$ satisfying

$$
s u=u \lambda_{u}(s)
$$

(see Fig. 3). Then $\lambda_{u}: I \rightarrow R$ is a homomorphism of Lie groups. Comparing (3.1.2) and (3.1.4) we find

$$
r^{\sigma}(s ; y)=\lambda_{\sigma(y)}(s), \quad s \in I,
$$


and thus $s \mapsto r^{\sigma}(s ; y)$, when restricted to $s \in I$, is a group homomorphism. Let $\mathscr{I} \equiv \operatorname{Lie}(I)$ be the Lie algebra of $I$. It follows then that $\Lambda^{\sigma}$ restricted to $\xi \in \mathscr{I}$ is a Lie algebra homomorphism, which coincides with the derivative $\lambda_{\sigma(y)}^{\prime}$ of $\lambda_{\sigma(y)}$. We thus see that $\Lambda^{\sigma}$ restricted to $\mathscr{I}$ depends only on the action of $S$ on $U$, and not on the connection. What does depend on $\omega$ is the restriction of $\Lambda^{\sigma}$ to a complement, say $\mathscr{P}$, of $\mathscr{I}$ in $\mathscr{S}$. Write $\mathscr{S}=\mathscr{I}+\mathscr{P}$ with $\operatorname{Ad}(I) \mathscr{P} \subset \mathscr{P}$ (reductive decomposition), and define $\phi^{\sigma}(\cdot ; y)$ to be the restriction of $\Lambda^{\sigma}$ to $\mathscr{P}$. The field $\phi$ defined in this way will be later on interpreted as the Higgs field resulting from dimensional reduction of the Yang-Mills field.

Fig. 3

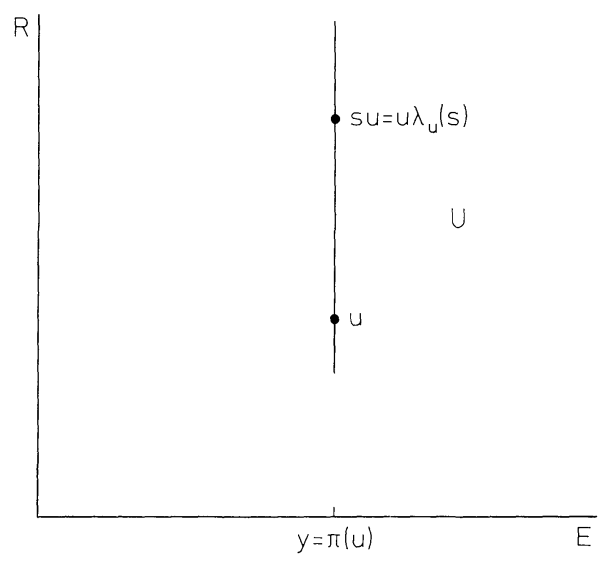

\subsection{Several Bundle Structures of the Principle Bundle $U$}

As before, consider a principal bundle $(U, E, \pi, R)$ with base $E$ and structure Lie group $R$, and let $S$ be a Lie group acting on $U$ by bundle automorphisms. We want to discuss now in more detail the structure arising from such an action. We will assume that both $R$ and $S$ are compact although, with proper care, our discussion could be carried through for non-compact groups admitting biinvariant, nondegenerate metric, with essentially the same results.

We recall from the previous subparagraph that there is an induced action of $S$ on the base $E$. For every $y \in E$ denote by $S_{y}$ the stability group of $y: S_{y}$ $=\{s \in S: s y=y\}$. There are then two natural equivalence relations in $E$. One is: $y \sim y^{\prime}$, if $S_{y}$ and $S_{y^{\prime}}$ are conjugate; the other, stronger one, is: $y \approx y^{\prime}$ iff $S_{y}=S_{y^{\prime}}$. The equivalence classes $[y]=\left\{y^{\prime} \in E: y^{\prime} \sim y\right\}$ are called strata. In general $E$ will be an union of several strata and, since $S$ is compact, one of them will be open and dense (see [22, 23]). Let us restrict our further discussion to one of these strata. Or, better, let us assume that $E$ consists of a single stratum only ${ }^{4}$. Thus $S_{y}$ and $S_{y^{\prime}}$ are conjugate for all $y, y^{\prime} \in E$. Consider now the second equivalence relation. The equivalence class $[[y]]=\left\{y^{\prime} \in E: y^{\prime} \approx y\right\}$ is conveniently called the substratum of $y$ [24]. We choose, once and for all, one of these substrata, call it $P$, and denote by $U$ the stability group common to all the points of $P: P=\left\{y \in E: S_{y}=I\right\}$. $P$ is a

$4 E$ is then called simple $S$-space and the action of $S$ on $E$ is called simple 

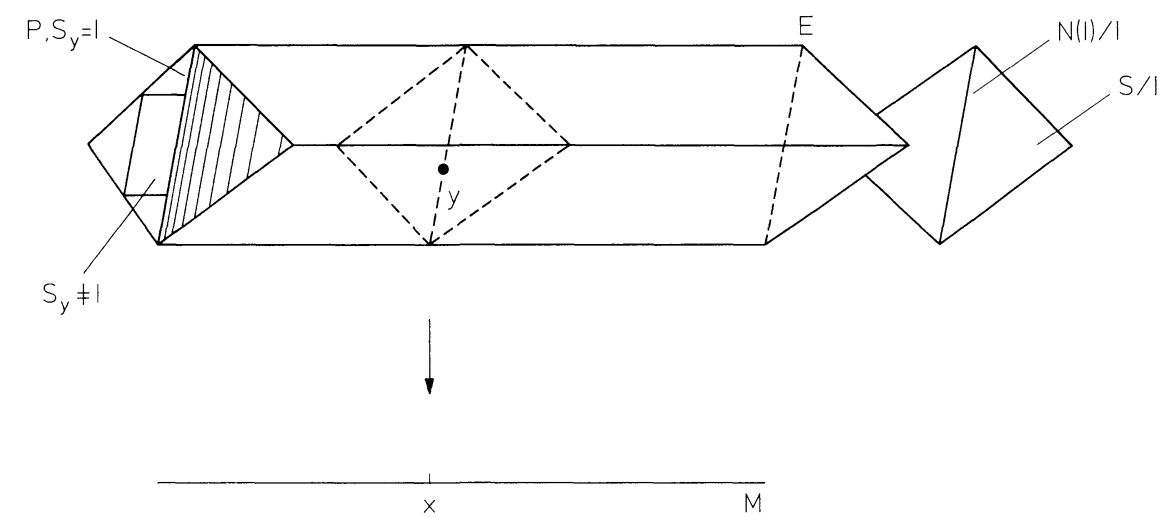

Fig. 4. Notice that $E=[y]$ and $P=[[y]] . P$ is a subbundle of $E$. The Killing vectors $Z_{\xi}, \xi \in \mathscr{S}$, introduced in Sect. 3.1 are vertical in $E$, those corresponding to $\xi \in \operatorname{Lie}(N(I))$ are, at the points of $P$, tangent to the submanifold $P$

submanifold of $E$, and $E$ can be thought of as a collection of orbits of type $S / I$, the collection being parametrized by a manifold $M$ - the manifold of orbits. In other words $E$ is a fiber bundle of base $M$ and fiber $S / I$ - see Fig. 4. It is important to realize that $P$ is a subbundle of $E$, it is a principal bundle with the same base $M$ and structure group $K \doteqdot N(I) / I, N(I)$ being the normalizer of $I$ in $S$ (see [22, 23, also 1]).

For our further discussion it is important to notice that the direct product group $G \equiv S \times R$ acts on $U$ via the following right action:

$$
G \equiv S \times R \ni(s, r): u \mapsto s^{-1} u r .
$$

With the terminology introduced above we will assume that this action is simple, i.e. that $U$ consists of a single stratum. To fix a substratum $Q$ of $U$ let us choose $u \in U$ such that $\pi(u) \in P$ [see Fig. 4] and let $H \equiv G_{u}$ be the stability group of this $u$. We take for the substratum $Q \subset U$ the substratum characterized by $H$ :

$$
Q=\left\{u \in U: s^{-1} u r=u \text { iff }(s, r) \in H\right\} .
$$

Since $Q=[[u]]$ and $\pi(u) \in P$, it follows that $\pi(Q) \subset P$. As before $U$ is a fiber bundle with (the same as $E$ ) base $M$ and fiber $G / H$, and $(Q, M, N(H) / H)$ is a principal bundle. The space $U$ can therefore be fibrated in several ways ${ }^{5}$ and Fig. 5 below summarizes the results.

In Sect. (3.1) we already introduced the group homomorphisms $\lambda_{u}: S_{\pi(u)} \rightarrow R$. Let us observe that $\lambda_{u}$ remains constant while $u$ varies in $Q$. Indeed, by the very definitions we have $G_{u}=\left\{\left(s, \lambda_{u}(s)\right): s \in S_{\pi(u)}\right\}$. When $u$ runs through $Q$, then $G_{u} \equiv H$ and $S_{\pi(u)} \equiv I$ are both constant, and thus $\lambda \equiv \lambda_{u}$ is constant too. Thus we have

$$
H=\{(i, \lambda(i)): i \in I\}=\operatorname{diag}(I, \lambda(I)) \subset G .
$$

The stability group $H$ is isomorphic to $I$, but it is not equal to $I x\{e\}$. Because of this fact the normalizer of $H$ in $G=S \times R$ is not isomorphic to $N(I) \times R$. Let us see what is the relation between $N(H)$ and $N(I)$. By using the definitions we find

$$
(s, r) \in N(H) \Leftrightarrow\left\{\begin{array}{l}
s \in N(I) \text { and } \\
\mathrm{r} \lambda(i) \mathrm{r}^{-1}=\lambda\left(\text { sis }^{-1}\right) \quad \forall i \in I .
\end{array}\right.
$$

5 Other fibrations are also possible but we will not need them here, see [22] 

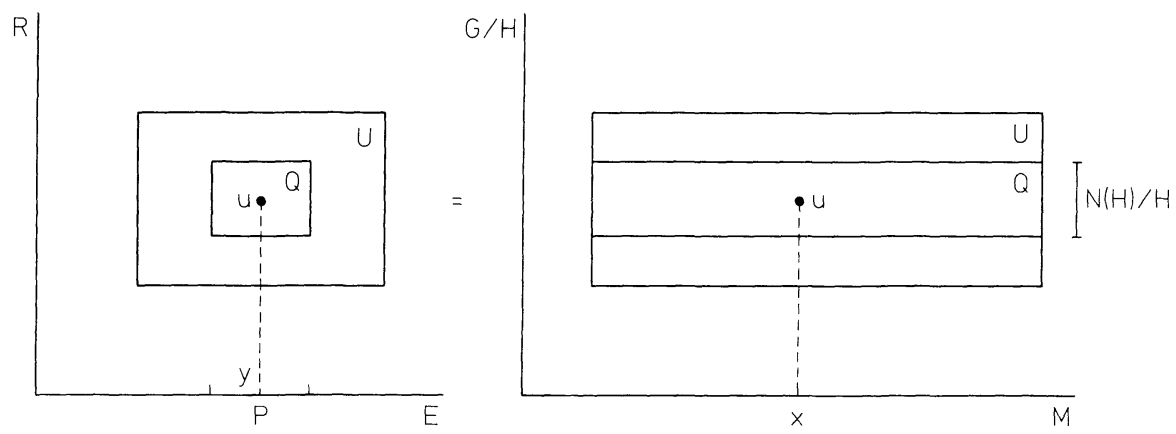

Fig. 5

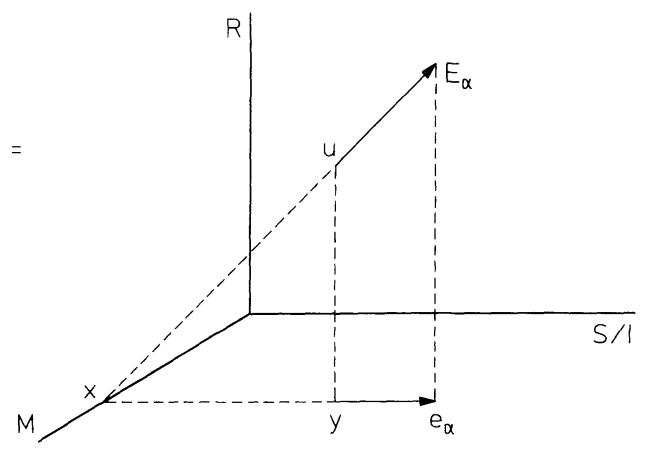

Consider the centralizer $Z$ of the image $\lambda(I)$ of $I$ in $R$ :

$$
Z \equiv\left\{r \in R: r \lambda(i) r^{-1}=\lambda(i), \forall i \in I\right\} \text {. }
$$

By the embedding $Z \rightarrow Z \times\{e\}, Z$ can be considered as a subgroup of $N(H) \mid H$, and one easily gets the following important result [8].

Proposition 3.2.1. $Z$ is an invariant subgroup of $N(H) \mid H$, and $N(H) \mid H$ is locally isomorphic to $(N(I) \mid I) \times Z, N(I)$ being the normalizer of $I$ in $S$.

\subsection{Lie Algebra Decomposition and the Vielbein}

We introduce the Lie algebra $\mathscr{S}, \mathscr{I}, \mathscr{N}(I), \mathscr{K}$ of $S, I, N(I), K \doteqdot N(I) \mid I$, and decompose the Lie algebra $\mathscr{S}$ of $S$ as follows (cf. [1,8]):

$$
\begin{gathered}
\mathscr{S}=\underbrace{\mathscr{I}+\overbrace{\mathscr{K}}+\mathscr{L}}_{\mathscr{N}(I)}, \quad \mathscr{P} \cong \mathscr{P}|\mathscr{I}, \quad \mathscr{K}=\mathscr{N}(I)| \mathscr{I}, \\
\mathscr{K}=\{\xi \in \mathscr{P}:[\xi, \mathscr{I}]=0\}, \quad[\mathscr{I}, \mathscr{K}]=0, \\
{[\mathscr{K}, \mathscr{K}] \subset \mathscr{K}, \quad[\mathscr{N}(I), \mathscr{L}] \subset \mathscr{L}, \quad[\mathscr{I}, \mathscr{P}] \subset \mathscr{P},}
\end{gathered}
$$

and let us introduce the Lie algebra bases $T_{\underline{\alpha}}, T_{\hat{\alpha}}, T_{\alpha}, T_{\hat{i}}, T_{i}$ in $\mathscr{I}, \mathscr{K}, \mathscr{P}, \mathscr{Z}$, and $\mathscr{R}$ respectively - see Fig. 6.

The homomorphism $\lambda: I \rightarrow R$ introduced in 3.2 induces the homomorphism $\lambda^{\prime}: \mathscr{I} \rightarrow \mathscr{R}$ of Lie algebras, and we define the matrix elements $\lambda_{\underline{\alpha}}^{i}$ of $\lambda^{\prime}$ by

$$
\lambda^{\prime}\left(T_{\underline{\alpha}}\right)=\lambda_{\underline{\alpha}}^{i} T_{i} .
$$



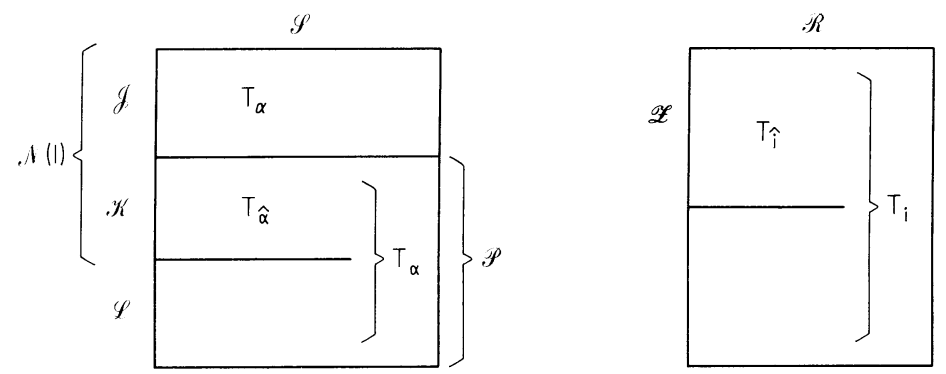

Fig. 6. Decompositions of the Lie algebras $\mathscr{S}$ and $\mathscr{R} . \mathscr{Z}$ denotes the Lie algebra of the centralizer $Z$ of $\lambda(I)$

The Lie algebra $\mathscr{G}$ of the direct product group $G=S \times R$ can be decomposed in two ways

where

$$
\mathscr{G}=\overbrace{\mathscr{I}+\mathscr{K}+\mathscr{L}}^{\mathscr{S}}+\mathscr{R}=\mathscr{H}+\overbrace{\mathscr{K}+\mathscr{L}}^{\mathscr{P}}+\mathscr{R},
$$

$$
\mathscr{H}=\left\{\xi+\lambda^{\prime}(\xi): \xi \in \mathscr{I}\right\}=\operatorname{diag}\left(\mathscr{I}, \lambda^{\prime}(\mathscr{I})\right) \subset \mathscr{S}+\mathscr{R}
$$

is the Lie algebra of the stability group $H$. It is the second decomposition which is used in the reduction theorem for $G / H$. To apply this theorem we have to introduce a new basis in $\mathscr{G}$, with tilda, adapted to the second, reductive, decomposition:

$$
\begin{aligned}
& \left.\begin{array}{ll}
\tilde{T}_{\alpha}=T_{\alpha}+\lambda^{\prime}\left(T_{\alpha}\right)(\mathscr{H}) \\
\tilde{T}_{\alpha}=T_{\alpha} & (\mathscr{P})
\end{array}\right\}, \quad \tilde{T}_{A} \equiv T_{A} \quad(\mathscr{G} / \mathscr{H}) \\
& \widetilde{T}_{i}=T_{i} \quad(\mathscr{R}), \quad \widetilde{T}_{\underline{A}} \equiv \widetilde{T}_{\alpha} \quad(\mathscr{H}) .
\end{aligned}
$$

It is important to notice that the Lie algebra of $N(H) \mid H$ is composed of $\mathscr{K}$ and $\mathscr{Z}$ (see Proposition 3.2.1, also [8])

$$
\operatorname{Lie}(N(H) \mid H)=\mathscr{K}+\mathscr{Z} .
$$

We shall use the index $\hat{A}=(\hat{\alpha}, \hat{i})$ to label generators of this algebra. Thus

$$
\tilde{T}_{\hat{A}}=T_{\hat{A}}=\left(T_{\hat{\alpha}}, T_{\hat{i}}\right) .
$$

The structure constants of the adapted (non-product) basis in $\mathscr{G}=\mathscr{S}+\mathscr{R}$, which differ from those of the product (non-tilda) basis, are the following ones

$$
\begin{aligned}
& \widetilde{C}_{\alpha \beta}^{i}=-C_{\alpha \alpha}^{\gamma} \lambda_{\underline{\underline{\nu}}}^{i}, \\
& \tilde{C}_{\alpha \underline{\beta}}^{i}=C_{j k}^{i} \lambda_{\underline{\alpha}}^{j} \lambda_{\underline{\beta}}^{k}-C_{\underline{\alpha} \underline{\beta}}^{\gamma} \lambda_{\underline{\underline{\gamma}}}^{i}, \\
& \tilde{C}_{\underline{\alpha} i}^{j}=\lambda_{\underline{\alpha}}^{k} C_{k i}^{j} .
\end{aligned}
$$

Observe that

$$
\tilde{C}_{\alpha \hat{\beta}}^{i}=C_{\alpha \hat{\beta}}^{i}=0 .
$$

Let now $g_{U}$ be a $G$-invariant metric on $U$. In particular, being $S$-invariant, $g_{U}$ induces a metric $g_{E}$ on $E$ (Sect. 2.2), and $g_{E}$ is $S$-invariant. Thus $g_{E}$ induces a metric $g_{M}$ on $M$. We recall that $M=E / S=U / G$, therefore $g_{M}$ can be also induced directly from $g_{U}$. For our purpose it will be enough to assume that $g_{U}$, restricted to the fibers 
of the principal bundle $U \rightarrow E$, induces a fixed biinvariant metric $k$ on $R$. We will call it the Killing metric.

Let $u$ be a point in $Q, y$-its projection in $P$, and let $x$ be the projection of $y$ on $M$ (see Fig. 5). We introduce the following vector fields:

$\partial_{\mu}-$ a holomorphic moving frame (vierbein) around $x \in M$,

$e_{\mu}$ - the horizontal, i.e. orthogonal to the fibers of $E \rightarrow M$, lift of $\partial_{\mu}$,

$e_{\alpha}$ - fundamental fields $e_{\alpha} \equiv Z_{T_{\alpha}}$ in $E$,

$e_{M}=\left(e_{\mu}, e_{\alpha}\right)-$ the vielbein around $y$,

$E_{\alpha}$ - fundamental fields $E_{\alpha} \equiv Z_{T_{\alpha}}$ in $U$,

$E_{i}$ - fundamental fields $E_{i} \equiv Z_{T_{i}}$ in $U$,

$E_{A}=\left(E_{\alpha}, E_{i}\right)-$ vertical vielbein around $u$ in $U$,

$E_{\hat{A}}=\left(E_{\hat{\alpha}}, E_{\hat{i}}\right)-$ vertical vielbein around $u$ in $Q$,

$\hat{E}_{\alpha}$ - the horizontal, i.e. orthogonal to the fibers of $U \rightarrow E$, lift of $e_{\alpha}$.

The components of the fields appearing in the following discussion will refer to the vielbeins introduced above. In particular we will use the following notation (some of the formulas will be explained later):

$$
\begin{aligned}
& g_{A B} \equiv g_{U}\left(E_{A}, E_{B}\right)=\left(g_{\alpha \beta}, g_{\alpha i}, g_{i j}\right), \quad g^{A B} \equiv\left(\text { the inverse of } g_{A B}\right), \\
& h_{M N} \equiv g_{E}\left(E_{M}, E_{N}\right)=\left(h_{\mu v}, h_{\alpha \beta}\right), \quad h^{M N} \equiv\left(\text { the inverse of } h_{M N}\right) \text {, } \\
& h_{\alpha \beta} \equiv g_{E}\left(e_{\alpha}, e_{\beta}\right)=g_{U}\left(\hat{E}_{\alpha}, \hat{E}_{\beta}\right), \quad h^{\alpha \beta} \equiv\left(\text { the inverse of } h_{\alpha \beta}\right) \text {, } \\
& \gamma_{\mu \nu} \equiv g_{M}\left(\partial_{\mu}, \partial_{v}\right)=g_{E}\left(e_{\mu}, e_{v}\right)=h_{\mu \nu}, \quad \gamma^{\mu v} \equiv\left(\text { the inverse of } \gamma_{\mu \nu}\right) \text {. }
\end{aligned}
$$

Let $\omega$ be the principal connection form on $U$ induced by $g_{U}$ (considered as $R$-invariant, see Sect. 2.1).

We define the fields $\phi_{\alpha}^{i}$ by

$$
\omega\left(E_{\alpha}(u)\right)=-\phi_{\alpha}^{i}(u) T_{i} .
$$

Then one easily finds the following relations

$$
\begin{gathered}
E_{\alpha}=\hat{E}_{\alpha}-\phi_{\alpha}^{i} E_{i} \\
g_{\alpha \beta}=h_{\alpha \beta}+\phi_{\alpha}^{i} \phi_{\beta}^{j} k_{i j}, \quad g^{\alpha \beta}=h^{\alpha \beta}, \\
g_{\alpha j}=-\phi_{\alpha}^{i} k_{i j}, \quad g^{\alpha i}=h^{\alpha \beta} \phi_{\beta}^{i}, \\
g_{i j}=k_{i j}, \quad g^{i j}=k^{i j}+\phi_{\alpha}^{i} \phi_{\beta}^{j} h^{\alpha \beta},
\end{gathered}
$$

where $k_{i j}$ are the components of the Killing metric of $R$. The $G=S \times R$-invariance of the metric $g_{U}$ implies that $\phi=\left(\phi_{\alpha}^{i}\right)$, considered as a linear map from $\mathscr{P}$ to $\mathscr{R}$, satisfies the constraint of $\operatorname{Ad}(H)$-invariance

$$
\phi \circ \operatorname{Ad}(i)=\operatorname{Ad}(\lambda(i)) \circ \phi, \quad i \in I,
$$

or, infinitesimally,

$$
C_{\underline{\alpha} \beta}^{\gamma} \phi_{\gamma}^{k}=\lambda_{\underline{\alpha}}^{i} \phi_{\beta}^{j} C_{i j}^{k}
$$

In particular $\phi_{\hat{\alpha}}^{i}=0$ if $i \neq \hat{i}$ and $\phi_{\alpha}^{\hat{i}}=0$ if $\alpha \neq \hat{\alpha}$, which means that $\phi(\mathscr{K}) \subset \mathscr{Z}$ and that $\phi(\mathscr{L}) \cap \mathscr{Z}=\{0\}$.

We also have

$$
C_{\underline{\alpha} \beta}^{\gamma} h_{\gamma \delta}+C_{\underline{\alpha} \delta}^{\gamma} h_{\beta \gamma}=0,
$$

which expresses the $I$-invariance of $h_{\alpha \beta}$. 


\section{Reduction of the Einstein Yang-Mills Action}

\subsection{Outline of the Method}

As was already explained in the introduction, our aim is to investigate dimensional reduction of $S$-invariant Einstein-Yang-Mills fields in a multidimensional universe. Thus we start with an $S$-invariant metric $g_{E}$ on $E$, and an $S$-invariant principal connection $\omega$ on $U$. We also fix a biinvariant metric $k_{i j}$ on the initial gauge group $R$. The logic followed in this section is summarized by the Tables 1 and 2 .

Table 1 stresses the equivalence between three possible descriptions of the same geometrical structure (we called them Description 1,2, and 3 in our introduction). The links between any two boxes of this chart are provided by the use of the Reduction Theorem (recalled in Sect. 2). The main goal of our work is to connect Description 2 and 3 (Link No. 1), and this will be done via Description 1.

Table 2 summarizes the formulae associated to the links $L 1, L 2, L 3, L 4, L 5$ of Table 1 via the use of the Reduction Theorem; all these relations are of course obtained and analyzed in the subsequent paragraphs. We introduce the following notations: YM (base, group) denotes the lagrangian for a Yang-Mills field defined on the space "base," valued in the Lie-algebra of "group"; KE (base, fiber) denotes the kinetic energy term containing "base derivatives" [Eqs. (3.1) and (3.4)] of the metric on the space "fiber"; finally, the scalar curvature of a space $F$ is denoted by $\tau_{F}$ or $\tau(F)$.

\subsection{The Link No. 2}

We start with Description 2: a multidimensional Universe $E$ furnished with an $S$-invariant metric $g(E)=\left(h_{M N}\right)$ and an $S$-invariant Yang-Mills field $A_{N}^{i}$ with values in the Lie algebra $\mathscr{R}$ of $R$; the $S$-invariance was discussed in Sect. 3.1. The EinsteinYang-Mills Lagrangian is given by the expression

$$
\operatorname{EYM}(E)=\tau(E)-\frac{1}{4} k_{i j} h^{M o} h^{N P} F_{M N}^{i} F_{O P}^{j},
$$

where $\tau(E)$ is the scalar curvature of $E$ for the metric $h_{M N}$, and $F_{M N}^{i}$ is the YangMills field strength associated to $A_{N}^{i}$. Provided that we add to this expression a constant $\tau(R)$ with value equal to the scalar curvature of the group $R$ (endowed with the Killing metric) we can use the Reduction Theorem of Sect. 2.1 to construct, out of these three pieces, an $R$-invariant metric $g(U)$ on $U$ considered as an $R$-principal bundle over $E$. This metric $g(U)$ will be actually $S \times R$-invariant because we started with the ingredients which were themselves $S$-invariant (here one also exploits biinvariance of the Killing metric $k$ ). The scalar curvature of $U$, associated to this metric $g(U)$ is

where

$$
\tau(U)_{u}=\tau(E)_{y}+\tau(R)+\mathrm{YM}(E, M)_{y},
$$

$$
\mathrm{YM}(E, R)_{y}=-\frac{1}{4} k_{i j} h^{M O} h^{N P} F_{M N}^{i}(y) F_{O P}^{j}(y),
$$

$u$ being any point in the $R$-fiber of $U$ over $y$. This geometrical structure, described in terms of the space $U$, constitutes what we called Description 1 in our introduction. 


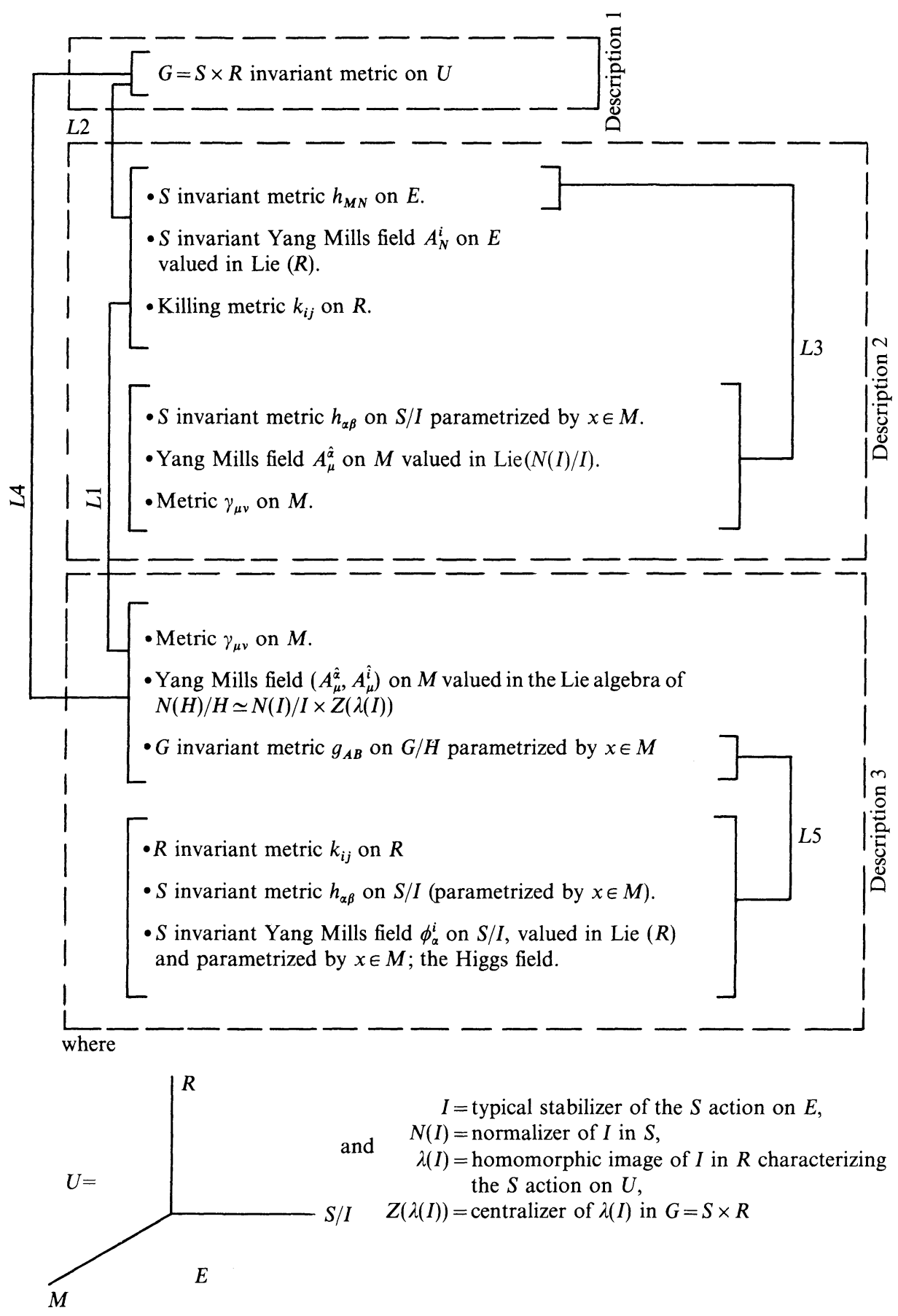


Table 2
L2: $\tau_{(U)}=\tau_{(E)}+\tau_{(R)}+\mathrm{YM}(E, R)$,
$L 3: \tau_{(E)}=\tau_{(M)}+\tau_{(S / I)}+\mathrm{YM}(M, N(I) \mid I)+\mathrm{KE}(M, S / I)$,
L4: $\tau_{(U)}=\tau_{(M)}+\tau_{(G / H)}+\mathrm{YM}(M, N(H) \mid H)+\mathrm{KE}(M, G / H)$,
L5: $\tau_{(G / H)}=\tau_{(S / I)}+\tau_{(R)}+\mathrm{YM}(S / I, R)$.

Also

$\mathrm{YM}(M, N(H) \mid H)=\mathrm{YM}(M, N(I) \mid I)+\mathrm{YM}(M, Z(\lambda(I))+\Delta$ cf. Eqs. (4.4.2)-(4.4.5),

$\mathrm{YM}(S / I, R)=-V(\lambda, \phi)$ cf. Eqs. (4.5.3) and (4.5.4),

$\mathrm{KE}(M, G / H)=\mathrm{KE}(M, S / I)+\mathrm{KE}(\phi)$ cf. Eqs. (4.3.5) and (4.4.6)

\subsection{The Link No. 3}

This link is a standard application of the Reduction Theorem of Sect. 2.2 to a $S$-invariant metric $g(E)$ on $E$. For the scalar curvature of $E$, endowed with this metric, we get [see (2.2.1) and (2.2.2)]:

$$
\tau(E)=\tau(M)+\tau(S / I)+\mathrm{YM}(M, N(I) \mid I)+\mathrm{KE}(M, S / I),
$$

where, with the notation given in Sect. 3.3,

$$
\begin{gathered}
R(M)=R\left(\gamma_{\mu \nu}\right), \\
R(S / I)=-h^{\alpha \alpha^{\prime}}\left(\frac{1}{2} C_{\alpha \beta}^{\gamma} C_{\alpha^{\prime} \gamma}^{\beta}+\frac{1}{4} h^{\beta \beta^{\prime}} h_{\gamma \gamma^{\prime}} C_{\alpha \beta}^{\gamma} C_{\alpha^{\prime} \beta^{\prime}}^{\gamma^{\prime}}+C_{\alpha \beta}^{\bar{\gamma}} C_{\alpha^{\prime} \underline{\gamma}}^{\beta}\right), \\
\operatorname{YM}(M, N(I) \mid I)=-\frac{1}{4} h_{\hat{\alpha} \hat{\alpha}^{\prime}} \gamma^{\mu \mu^{\prime}} \gamma^{v v^{\prime}} F_{\mu \nu}^{\hat{\alpha}} F_{\mu^{\prime} \nu^{\prime}}^{\hat{\alpha}^{\prime}}, \\
\mathrm{KE}(M, S / I)=-\frac{1}{4} h^{\alpha \beta} h^{\gamma \delta}\left(D_{\mu} h_{\alpha \gamma} D^{\mu} h_{\beta \delta}+D_{\mu} h_{\alpha \beta} D^{u} h_{\gamma \delta}\right) \\
-\nabla_{\mu}\left(h^{\alpha \beta} D^{\mu} h_{\alpha \beta}\right) .
\end{gathered}
$$

\subsection{The Link No. 4}

As explained in Sect. 3.2, $U$ is also a $G / H$ bundle over $M$ (recall that, since $U \simeq E \times R$, the manifold $M$ of $G=S \times R$-orbits in $U$ is the same as the manifold of $S$-orbits in $E$ ); here again we can use the Reduction Theorem. The $G$-invariant metric $g(U)$ on $U$ can be expressed as being built out of the following three pieces: a metric $g(M)=\left(\gamma_{\mu \nu}\right)$ on $M$ (usually interpreted as space-time metric), a Yang-Mills field $A_{\mu}^{\mathscr{A}}$ valued in Lie $(N(H) / H)=\mathscr{K}+\mathscr{Z}$ [see (3.3.2)], and a scalar field $h_{A B}(x)$ which can be interpreted as a $G$-invariant metric in the internal space $U_{x} \simeq G / H$ above $x \in M$. The scalar curvature $\tau(U)$ of $U$ can now be written entirely in terms of $M$-based quantities:

$$
\tau_{u}(U)=\tau_{x}(M)+\tau_{x}(G / H)+\mathrm{YM}(M, N(H) \mid H)+\mathrm{KE}(M, G / H),
$$

where

$$
\begin{aligned}
& \tau(G / H)=-g^{A A^{\prime}}\left(\frac{1}{2} \widetilde{C}_{A B}^{C} \widetilde{C}_{A^{\prime} C}^{B}+\frac{1}{4} g^{B B^{\prime}} g_{C C^{\prime}} \widetilde{C}_{A B}^{C} \widetilde{C}_{A^{\prime} B^{\prime}}^{C^{\prime}}+\widetilde{C}_{\bar{A} B}^{C} \widetilde{C}_{A^{\prime} \underline{C}}^{B}\right), \\
& \mathrm{YM}(M, N(H) \mid H)=-\frac{1}{4} \gamma^{\mu \mu^{\prime}} \gamma^{\nu v^{\prime}} g_{\hat{A} \hat{B}} F_{\mu \nu}^{\hat{A}} F_{\mu^{\prime} v^{\prime}}^{\hat{B}}, \\
& \mathrm{KE}(M, G / H)=-\frac{1}{4} \gamma^{\mu v} g^{A B} g^{C D}\left(\tilde{D}_{\mu} g_{A C} \tilde{D}_{v} g_{B D}+\tilde{D}_{\mu} g_{A B} \tilde{D}_{v} g_{C D}\right) \text {, }
\end{aligned}
$$


and

$$
\tilde{D}_{\mu} g_{A B}=\partial_{\mu} g_{A B}+\tilde{C}_{A \hat{C}}^{D} A_{\mu}^{\hat{C}} g_{D B}+\tilde{C}_{B \hat{C}}^{D} A_{\mu}^{\hat{C}} g_{A D} .
$$

These formulae may be understood as referring to a certain local cross-section

$$
\sigma: M \ni x \rightarrow \sigma(x) \in Q \subset U .
$$

The tildas refer to the reductive basis in $\mathscr{G}$ (3.3.1). To reduce further the above expressions we apply the relations (3.3.3) and (3.3.7) with the result

$$
\mathrm{YM}(M, N(H) \mid H)=\mathrm{YM}(M, K)+\mathrm{YM}(M, Z)+\Delta,
$$

where

$$
\begin{aligned}
& \mathrm{YM}(M, K)=-\frac{1}{4} \gamma^{\mu \mu^{\prime}} \gamma^{v v^{\prime}} h_{\hat{\alpha} \hat{\beta}} F_{\mu \nu}^{\hat{\alpha}} F_{\mu^{\prime} v^{\prime}}^{\hat{\beta}}, \\
& \mathrm{YM}(M, Z)=-\frac{1}{4} \gamma^{\mu \mu^{\prime}} \gamma^{v v^{\prime}} k_{\hat{i} \hat{j}} F_{\mu \nu}^{\hat{i}} F_{\mu^{\prime} v^{\prime}}^{\hat{j}}
\end{aligned}
$$

and

$$
\Delta=-\frac{1}{4} \gamma^{\mu \mu^{\prime}} \gamma^{\nu v^{\prime}} k_{\hat{i} \hat{j}} \phi_{\hat{\alpha}}^{\hat{i}} F_{\mu \nu}^{\hat{\alpha}}\left(\phi_{\hat{\beta}}^{\hat{i}} F_{\mu^{\prime} v^{\prime}}^{\hat{\beta}}-2 F_{\mu^{\prime} v^{\prime}}^{\hat{j}}\right) .
$$

We also get $\mathrm{KE}(M, G / H)=\mathrm{KE}(M, S / I)+\mathrm{KE}(\phi)$, where

$$
\mathrm{KE}(\phi)=-\frac{1}{2} h^{\alpha \beta} \gamma^{\mu v} k_{i j} D_{\mu} \phi_{\alpha}^{i} D_{\nu} \phi_{\beta}^{j},
$$

with

$$
D_{\mu} \phi_{\alpha}^{i}=\partial_{\mu} \phi_{\alpha}^{i}+C_{\alpha \hat{\gamma}}^{\delta} A_{\mu}^{\hat{\gamma}} \phi_{\delta}^{i}+C_{\hat{j} k}^{i} A_{\mu}^{\hat{j}} \phi_{\alpha}^{k} .
$$

Notice that when $K=N(I) / I$ [respectively $Z=Z(\lambda(I))]$ is discrete, then the $2^{\text {nd }}$ (respectively $3^{\text {rd }}$ ) term of (4.4.7) vanishes as well as (4.4.5). We omit the result one gets for $\tau(G / H)$ since it will be derived in a different way in the discussion of the Link No. 5 below.

\subsection{The Link No. 5}

The term $\tau_{x}(G / H)$ in (4.4.1) is the scalar curvature of the fiber $U_{x}$ of the bundle $(U, M, G / H)$. It is easy to see that the projection $\pi: U \rightarrow E$ makes $U_{x} \simeq G / H$ into a principal bundle with base $E_{x} \simeq S / I$ and structure group $R$ (Fig. 7):

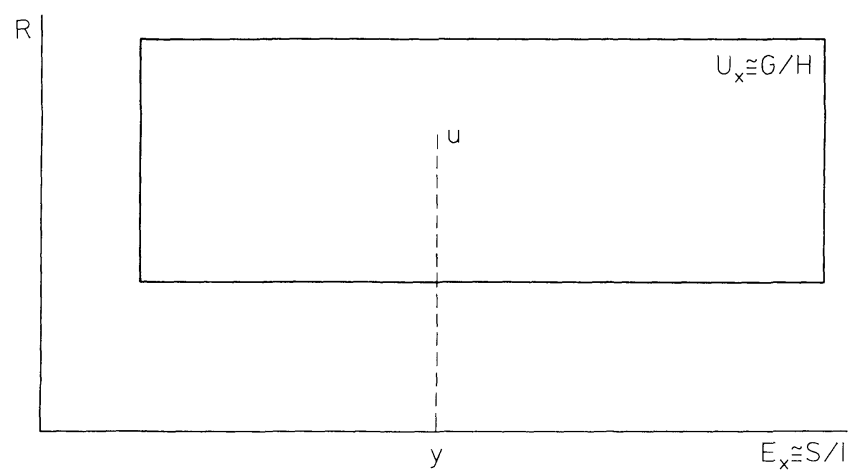

Fig. 7 
The metric $g_{A B}$ [see (3.3.5)] is $S \times R$-invariant on $U_{x}$ and therefore, a fortiori, $R$-invariant. Therefore we can apply the Reduction Theorem in its principal bundle version (Sect. 2.1): $g_{A B}$ can be expressed entirely in terms of an (S-invariant) metric $h_{\alpha \beta}$ on $E_{x}$, an (S-invariant) Yang-Mills field $\phi_{\alpha}^{i}$ on $E_{x}$, valued in $\mathscr{R}$, and the metric $k_{i j}$ on $R$ (the Killing metric). The explicit expression of $g_{A B}$ in terms of its building blocks has been already given in (3.3.7). Applying the Reduction Theorem of Sect. 2.1, we also get

$$
\tau(G / H)=\tau(S / I)+\tau(R)+\mathrm{YM}(S / I, R),
$$

where $\tau(R)$ and $\tau(S / I)$ already appeared in (4.2.2) and (4.3.1) respectively. Let us discuss now the Yang-Mills term. Since $S$ acts transitively on the base $E_{x} \simeq S / I$ of our bundle, we are in the situation of the Wang Theorem (see e.g. [25]), and we could simply refer to the literature. However, it is both instructive and convenient to obtain the desired expression directly. Denoting by $\omega=\left(\omega_{\alpha}^{i}\right)$ and $F=\left(F_{\alpha \beta}^{i}\right)$ the connection and curvature form of the induced Yang-Mills field, from the very definition of the curvature we get

$$
F_{\alpha \beta}=D \omega\left(E_{\alpha}, E_{\beta}\right)=E_{\alpha} \omega\left(E_{\beta}\right)-E_{\beta} \omega\left(E_{\alpha}\right)-\omega\left(\left[E_{\alpha}, E_{\beta}\right]\right)+\left[\omega\left(E_{\alpha}\right), \omega\left(E_{\beta}\right)\right],
$$

while the $S$-invariance of $\omega$ implies

$$
0=\left(L_{E_{\alpha}} \omega\right)\left(E_{\beta}\right)=E_{\alpha} \omega\left(E_{\beta}\right)-\omega\left(\left[E_{\alpha}, E_{\beta}\right]\right) .
$$

Combining the two formulae we get

$$
F_{\alpha \beta}=\omega\left(\left[E_{\alpha}, E_{\beta}\right]\right)+\left[\omega\left(E_{\alpha}\right), \omega\left(E_{\beta}\right)\right] .
$$

Now

$$
\omega\left(\left[E_{\alpha}, E_{\beta}\right]\right)=\omega\left(C_{\alpha \beta}^{\gamma} E_{\gamma}+C_{\alpha \beta}^{\gamma} E_{\underline{\gamma}}\right)=C_{\alpha \beta}^{\gamma} \omega\left(E_{\gamma}\right)+C_{\alpha \beta}^{\gamma} \omega\left(E_{\underline{\gamma}}\right),
$$

and we can use the formula (3.3.6) as well as the fact that $E_{\gamma}+\lambda_{\gamma}^{i} E_{i}$ vanishes on $Q$ [see (3.3.1)], to get

$$
\omega\left(\left[E_{\alpha}, E_{\beta}\right]\right)=-\left(C_{\alpha \beta}^{\gamma} \phi_{\gamma}^{i}+C_{\alpha \beta}^{\gamma} \lambda_{\underline{\gamma}}^{i}\right) T_{i}
$$

and consequently

$$
F_{\alpha \beta}^{i}=-\left(C_{\alpha \beta}^{\gamma} \phi_{\gamma}^{i}+C_{\alpha \beta}^{\gamma} \lambda_{\underline{\gamma}}^{i}-C_{j k}^{i} \phi_{\alpha}^{j} \phi_{\beta}^{k}\right) .
$$

The Yang-Mills term of (4.5.1) gives therefore the potential energy for the Higgs field $\phi$ :

$$
-V(\phi)=\mathrm{YM}(S / I, R)=-\frac{1}{4} h^{\alpha \alpha^{\prime}} h^{\beta \beta^{\prime}} k_{i j} F_{\alpha \beta}^{i} F_{\alpha^{\prime} \beta^{\prime}}^{j}
$$

with $F_{\alpha \beta}^{i}$ given by (4.5.3).

\subsection{The Link No. 1}

By simple collecting of the results obtained so far we find the following theorem.

Einstein-Yang-Mills Reduction Theorem. There is one-to-one correspondence between pairs $(g(E), A(E))$ of $S$-symmetric Einstein-Yang-Mills systems on $E$ and the 
quadruples $(g(M), A(M), \phi, h)$ of fields on $M$. The Einstein-Yang-Mills Lagrangian $\operatorname{EYM}(E)$ of $E$, when expressed in terms of the component fields on $M$ reads

$$
\operatorname{EYM}(E)=\tau(\gamma)+\operatorname{YM}\left(A_{\mu}^{\hat{i}}, A_{\mu}^{\hat{\alpha}}\right)+\operatorname{KE}\left(h_{\alpha \beta}\right)+\operatorname{KE}\left(\phi_{\alpha}^{i}\right)-V(h)-V(\phi),
$$

where

$\tau(\gamma)$ is the scalar curvature of the metric $g(M)=\left(\gamma_{\mu \nu}\right)$,

$\mathrm{YM}\left(A_{\mu}^{\hat{i}}, A_{\mu}^{\hat{\alpha}}\right)=\mathrm{YM}(M, N(H) / H)$ is given by $(4.4 .2)-(4.4 .5)$,

$\mathrm{KE}\left(h_{\alpha \beta}\right)=\mathrm{KE}(M, S / I)$ is given by (4.3.5),

$\mathrm{KE}\left(\phi_{\alpha}^{i}\right)=\mathrm{KE}(\phi)$ is given by (4.4.6), (4.4.7),

$V(h)=\tau(S / I)$ is given by (4.3.3),

$V(\phi)$ is given by (4.5.4).

As a by-product we also get the reduction formulae for the Yang-Mills term alone:

$$
\mathrm{YM}(E, R)=\mathrm{YM}(M, Z(\lambda(I))+\mathrm{KE}(\phi)+\Delta-V(\phi),
$$

where, however, the terms $\operatorname{KE}(\phi)$ and $\Delta$ depend on the connection $A_{\mu}^{\hat{\alpha}}$ in the $(P, M, K)$ bundle too.

One has to remember that the scalar fields $\phi_{\alpha}^{i}$ and $h_{\alpha \beta}$ satisfy the algebraic constraints (3.3.8) and (3.3.9).

Remark 4.6.1. The expressions (4.6.1) and (4.6.2) can be still multiplied by $\left|\operatorname{det} h_{\alpha \beta}\right|^{1 / 2}$, compare Remark 2.1.1.

\section{Examples, Comments, and Summary}

\subsection{Summary of the Results}

Space-time is, in this paper, identified with the manifold $M$ of orbits of a compact group $S$ (global symmetry group) acting on a manifold $E$ (extended space-time, multidimensional universe). Thus each space-time point $x \in M$ has internal structure of a homogeneous space $S / I$. $I$ is the isotropy group characterizing the orbit of $S$ over $x$.

Let $g(E), A(E)$ be an Einstein-Yang-Mills system in $E$, consisting of a (pseudo) Riemannian metric $g(E)$ and a Yang-Mills field $A(E)$ on $E$ with gauge group $R$. We show how such a system can be interpreted in terms of fields on $M$, when a constraint of $S$-symmetry is imposed. It is proved that there is a one-to-one correspondence between $S$-invariant Einstein-Yang-Mills systems $(g(E), A(E))$ and quadruples $(g(M), A(M), \phi, h)$, where $g(M)$ is a metric on $M, A(M)$ is a YangMills field on $M$ with the effective gauge group $N(H) / H$ described below, while $\phi$ and $h$ are scalar fields. $g(M)$ and $h$ originate from $g(E), \phi$ originates from $A(E)$, while $A(M)$ takes its origin from both $g(E)$ and $A(E)$.

The effective gauge group is the quotient $N(H) / H$, where $N(H)$ is the normalizer of $H$ in $G \equiv S \times R$, and $H \subset G$ is defined as $H=\operatorname{diag}(I \times \lambda(I)), \lambda: I \rightarrow R$ being the group homomorphism determined by the action of symmetry group $S$ on the gauge field (see below). Locally $N(H) / H$ is isomorphic to the product $(N(I) / I) \times Z$, where $N(I)$ is the normalizer of $I$ in $S$, and $Z$ is the centralizer of $\lambda(I)$ in $R$. 
The homomorphism $\lambda: I \rightarrow R$, where $I$ is the stability subgroup of $S$ at $y \in E$, is defined by the action of $S$ on the principal $R$-bundle $U$ over $E$ on which the initial gauge field $A(E)$ lives. Let $u \in U$ be such that $\pi(u)=y$. Then for each $s \in I, s u=u \lambda(s)$.

The derivative $\lambda^{\prime}: \mathscr{I} \rightarrow \mathscr{R}$ of $\lambda$ is a homomorphism of Lie algebras. $\lambda^{\prime}$ and $\phi$ are parts of the map $\Lambda: \mathscr{S} \rightarrow \mathscr{R}$ defined as follows: every $\xi \in \mathscr{S}$ is an infinitesimal symmetry of $A_{E}$. Thus the Lie derivative $L_{\xi} A_{E}$ is an infinitesimal gauge transformation - there exists a function $\Lambda_{y}: \xi \rightarrow \Lambda_{y}(\xi) \in \mathscr{R}$ such that $L_{\xi} A_{E}$ $=D \Lambda(\xi)$. For $\xi \in I, \Lambda(\xi)=\lambda^{\prime}(\xi)$. On the other hand write $\mathscr{S}=\mathscr{I}+\mathscr{P}$ with $\mathscr{P}$ such that $\operatorname{Ad}(I) \mathscr{P} \subset \mathscr{P}$. Then $\phi(\xi ; x)=\Lambda_{y}(\xi)$ for $x=\pi(y)$. Thus $\phi(x)$ is a linear map $\phi(x): \mathscr{P} \rightarrow \mathscr{R}$. It is constrained to satisfy

$$
\phi \circ \operatorname{Ad}(s)=\operatorname{Ad}(\lambda(s)) \circ \phi, \quad s \in I .
$$

The field $h$, originating from $g_{E}$, describes an $S$-invariant metric on the homogeneous space $S / I$ at $x$; algebraically $h=\left(h_{\alpha \beta}\right)$ is an $\operatorname{Ad}(I)$-invariant scalar product on $\mathscr{P}$.

The Einstein-Yang-Mills lagrangian for $\left(g_{E}, A_{E}\right)$ on $E$ when represented in terms of $(g(M), A(M), \phi, h)$ is given in Eq. (4.6.1). The field $\phi$ splits into two parts $\phi_{\mathscr{K}}$ and $\phi_{\mathscr{L}}$, where $\mathscr{K}$ is the space of $I$-singlets in $\mathscr{P}$ and $\mathscr{L}$ is a complement of $\mathscr{K}$ in $\mathscr{S}$. The fields $h$ and $\phi_{\mathscr{L}}$ interact with $A(M)$ by the minimal interaction while $\phi_{\mathscr{K}}$ interacts also directly with the Yang-Mills strength. The potential energy term for $\phi_{\mathscr{K}}$ and $\phi_{\mathscr{L}}$ is quartic.

The results improve those of [19-21] - the new ingredient is the part $N(I) / I$ of the gauge group (with Lie algebra isomorphic to $\mathscr{K}$ ) which may be present if $S / I$ is not isotropy irreducible.

\subsection{The Space of Higgs Fields $\phi$}

Let us first remember that we have a linear map $\Lambda$ from $\mathscr{S}=\mathscr{I}+\mathscr{K}+\mathscr{L}=\mathscr{I}+\mathscr{P}$ into $\mathscr{R}=\operatorname{Lie}(R)$ and that $\Lambda$ has to coincide on the subalgebra $\mathscr{I}$ of $\mathscr{S}$ with the algebra homomorphism $\lambda^{\prime}$ which characterizes the $S$ action. The Higgs field $\phi$ was defined as the restriction of $\Lambda$ to the complement $\mathscr{P}$ of $\mathscr{I}$ in $\mathscr{S}$; it is also convenient to split $\phi$ in $\phi=\phi_{\mathscr{K}}+\phi_{\mathscr{L}}$, where $\phi_{\mathscr{K}}=\left(\phi_{\hat{\alpha}}^{\hat{i}}\right)$ maps $\mathscr{K}$ into $\mathscr{Z} C \mathscr{R}$ and $\phi_{\mathscr{L}}$ maps $\mathscr{L}$ into a complement $W$ of $\mathscr{Z}$ in $\mathscr{R}$ (see also Sect. 3.3). Schematically:



where $\mathscr{N}\left(\lambda^{\prime}(I)\right)$ is the normalizer of $\lambda^{\prime}(I)$ in $\mathscr{R}, C\left(\lambda^{\prime}(I)\right)$ the center of $\lambda^{\prime}(I)$, and $W^{\prime}$ a supplementary subspace. 
It is easy to show that, under the action of the group $N(H)$, the map $\Lambda$ transforms as follows: let $(\sigma, \varrho) \in N(H) \subset S \times R$ and $s \in \mathscr{Y}$. Then

$$
\Lambda(s) \in \mathscr{R} \rightarrow \varrho^{-1} \Lambda\left(\sigma s \sigma^{-1}\right) \varrho \in \mathscr{R} .
$$

Moreover [see (3.3.8)], $\Lambda$ satisfies the constraint

$$
\Lambda\left(i s i^{-1}\right)=\lambda(i) \Lambda(s) \lambda(i)^{-1} \text { for } \quad i \in I .
$$

From this we can deduce the transformation properties of $\phi_{\mathscr{K}}$ and $\phi_{\mathscr{L}}$ under the gauge group $N(H) / H$ whose Lie algebra is $\mathscr{Z}+\mathscr{K}$; in particular:

- $K$ acts on $\phi_{\mathscr{K}}$ via the coadjoint representation, and $Z$ via the adjoint - this can also be seen from the fact that $\phi=\left(\phi_{\dot{\alpha}}^{\hat{i}}\right)$ has lower indices in $\mathscr{K}$ and upper indices in $\mathscr{Z}$. There are no constraints on $\phi_{\mathscr{K}}$ coming from (5.2.2),

- $K$ and $Z$ act as above on the space of $\phi_{\mathscr{L}}$ fields, however now $\phi_{\mathscr{L}}$ has to satisfy the constraint $\phi_{\mathscr{L}}\left(i s i^{-1}\right)=\lambda(i) \phi_{\mathscr{L}}(s) \lambda(i)^{-1}$ for $i \in I s \in \mathscr{S}$.

The representation of the gauge group on the space of $\phi$ fields is usually reducible; in order to find which irreducible representations appear, it is convenient to decompose $\mathscr{L}$ into irreducible representations of the product $I \cdot K$ by looking at the branching rule for ad $S$ into $I \cdot K$ and to decompose $W^{\prime}$ into irreducible representations of $\lambda(I) \cdot Z$ by looking at the branching rule for ad $R$ into $\lambda(I) Z$.

\subsection{Extrema of Potentials}

a) The potential $V(\phi)$ for the Higgs field can be written as the norm of the YangMills strength $F_{\alpha \beta}^{i}$ for a connection $\phi_{\alpha}^{i}$ on $G / H$ considered as a $R$-principal bundle over $S / I$ (see Sect. 4.5); $V(\phi)$ is therefore automatically invariant under $R$ and in particular under the subgroup $Z$.

Therefore the zeros of $V(\phi)$, which are at the same time absolute minima, are those maps $\phi$ which extend $\lambda$ to Lie algebra homomorphisms; this observation was already made in [21]. Then it is not too difficult to prove [using (5.2.1)] that the "unbroken" gauge group (the stability group of $\phi$ ) has Lie algebra isomorphic to $\mathscr{Z}_{\Lambda}+\mathscr{K}$, where $\mathscr{Z}_{\Lambda} \subset \mathscr{Z}$ is the commutant (centralizer) of $\Lambda(\mathscr{S})$ in $\mathscr{R}$, and $\mathscr{K}=\operatorname{Lie}(N(I) / I)$.

b) The potential $V(h)=-\tau(S / I)$ for the scalar fields $h_{\alpha \beta}(x)$ is more difficult to analyze. First, the scalar curvature $\tau(S / I)$ for the metric $h=\left(h_{\alpha \beta}\right)$ is not necessarily of a fixed sign (for example, it is not necessarily positive even if $S / I$ is compact and $h_{\alpha \beta}$ is positive definite). Next, it is well known that the saddle points of the functional $h \rightarrow \int_{S / I} \tau(S / I ; h) d \operatorname{vol}(h)$ when $h$ varies in the space of all metrics with fixed volume element, coincide with Einstein metrics on $S / I$ [29]. In many cases, however, saddle points of $\tau_{S / I}(h)$ when $h$ varies in the space of $S$-invariant metrics (with fixed volume) coincide also with $S$ invariant Einstein metrics on $S / I$. Notice that these saddle points are usually neither minima nor maxima. The potential $V(h)$ is clearly invariant under the whole group of diffeomorphisms of the differentiable structure of $S / I$; it is in particular invariant under the group $N(I) / I \times S$. All the metrics we are considering (all the fields $h_{\alpha \beta}$ ) are, by assumption, $S$-invariant; their full isometry group can of course be bigger; if $h_{\alpha \beta}^{0}$ is a saddle point 
of $V(h)$ and if the isometry group of $h^{0}$ is included in $N(I) / I \times S$ [it will then be of the kind $F \times S$, with $F \subset N(I) / I]$ we will say that the $N(I) / I$ piece of the gauge group is "broken" to $F$.

\subsection{Normalization and Units}

a) Sign Conventions. If $M$ is interpreted as a four dimensional space-time with signature -+++ , then, the Einstein lagrangian with cosmological term is $\frac{1}{16 \pi K}(\tau(M)-2 \Lambda)$. For positivity reasons, the signature of the "internal" metric on $S / I$ has to be spacelike, i.e., of positive sign with the above convention. Notice that the cosmological "constant" is in our case a function of $x \in M$; however, if we expand the internal metric $h_{\alpha \beta}$ around some background $h_{\alpha \beta}^{0}$ we obtain indeed a constant [coming from the $\tau(S / I)$ term] to be identified with the cosmological term; however, if $\tau^{0}(S / I) \equiv \tau\left(S / I ; h^{0}\right)$ is a positive scalar curvature, then $\Lambda$ will be negative and vice versa.

If we choose the signature +-- on $M$, the "physical" conclusions are of course the same, but one has to remember that in order to be spacelike, the signature of the "internal" metric $h_{\alpha \beta}$ has to be taken negative; the scalar curvature of a standard 2-sphere $S^{2}$, for example, would therefore also be negative. In what follows, we assume that our choice for $M$ is -+++ , therefore $h_{\alpha \beta}$ (and $k_{i j}$ ) are positive definite.

b) Dimensions. When computing the Einstein-Yang-Mills action on $E \simeq M \times S / I$ for a symmetric configuration of the metric and of the Yang-Mills field, we have to integrate over the "internal" space $S / I$. The integration itself is quite trivial (because of the symmetry under the $S$ group) and we obtain, at each point $x \in M$, the volume $V(x)$ of the "internal" space. This quantity $V(x)$ needs not be a constant unless we impose this new constraint. Let us however assume that, in the following, $V \equiv V(x)$ is constant; if this is not the case we will just have to multiply the final four-dimensional lagrangian by a real valued function on $M$ [compare Remarks (2.1.1) and (4.6.1)]. We start with the following dimensionless Einstein-Yang-Mills action:

$$
A=\int_{E}\left[\frac{1}{16 \pi \bar{K}} \tau(E)-\frac{1}{\bar{g}^{2}} \frac{1}{4} F_{M N}^{i} F_{M^{\prime} N^{\prime}}^{j} k_{i j} h^{M M^{\prime}} h^{N N^{\prime}}\right] d \operatorname{vol}(E),
$$

where $\bar{K}$ and $\bar{g}^{2}$ are a priori independent quantities. Let us then make the following changes (in that order):

1) Set $h_{\alpha \beta}=R^{2} \tilde{h}_{\alpha \beta}, R$ being some (length) scale.

2) Set $V=R^{n} \tilde{V}, \tilde{V}$ being the volume of $S / I$ for the metric $\tilde{h}$.

3) Define $\frac{1}{16 \pi K}=R^{n} \tilde{V} / 16 \pi \bar{K}$.

4) Define $1 / g_{s}^{2}=R^{n+2} \tilde{V} / 16 \pi \bar{K}$ and $1 / g_{R}^{2}=R^{n} \tilde{V} / \bar{g}^{2}$.

5) Rescale the fields $\widetilde{A}_{\mu}^{\hat{\alpha}}=\frac{1}{g_{s}} A_{\mu}^{\hat{\alpha}}, \tilde{A}_{\mu}^{\hat{i}}=\frac{1}{g_{R}} A_{\mu}^{\hat{i}}, \widetilde{\phi}_{\alpha}^{i}=\frac{1}{R g_{R}} \phi_{\alpha}^{i}$, and $\tilde{\lambda}_{\underline{\alpha}}^{i}=\frac{1}{R g_{R}} \lambda_{\underline{\alpha}}^{i}$. 
We find

$$
\begin{aligned}
& A=\int_{M}\left\{\frac{1}{16 \pi K} \tau_{M}+\frac{1}{16 \pi K R^{2}} \tilde{\tau}_{S / I}-\frac{1}{4} \widetilde{F}_{\mu \nu}^{\hat{\alpha}} \tilde{F}_{\mu^{\prime} v^{\prime}}^{\hat{\beta}} \gamma^{\mu \mu^{\prime}} \gamma^{\nu v^{\prime}} \tilde{h}_{\hat{\alpha} \hat{\beta}}\right. \\
& -\frac{1}{4} \tilde{F}_{\mu \nu}^{\hat{i}} \tilde{F}_{\mu^{\prime} v^{\prime}}^{\hat{j}} \gamma^{\mu \mu^{\prime}} \gamma^{\nu v^{\prime}} k_{\hat{i} \hat{j}}-\frac{1}{4}\left(R^{2} g_{R}^{2}\right) \tilde{\phi}_{\hat{\alpha}}^{i} \widetilde{\phi}_{\hat{\beta}}^{\hat{j}} \widetilde{F}_{\mu \nu}^{\hat{\alpha}} \tilde{F}_{\mu^{\prime} v^{\hat{\beta}}} \gamma^{\mu \mu^{\prime}} \gamma^{v v^{\prime}} k_{\hat{i} \hat{j}} \\
& -\frac{1}{2}\left(R g_{R}\right) \widetilde{\phi}_{\hat{\alpha}}^{\hat{i}} \tilde{F}_{\mu \nu}^{\hat{\alpha}} \tilde{F}_{\mu^{\prime} v^{\prime}}^{\hat{j}} k_{\hat{i} \hat{j}}-\frac{1}{4} \frac{1}{16 \pi K} \tilde{h}^{\alpha \beta} \tilde{h}^{\gamma \delta}\left[D_{\mu} \tilde{h}_{\alpha \beta} D^{\mu} \tilde{h}_{\gamma \delta}+D_{\mu} \tilde{h}_{\alpha \gamma} D^{\mu} \tilde{h}_{\beta \delta}\right] \\
& -\frac{1}{2} D_{\mu} \tilde{\phi}_{\alpha}^{i} D_{\mu}, \tilde{\phi}_{\beta}^{j} \tilde{h}^{\alpha \beta} k_{i j} \gamma^{\mu \mu^{\prime}}-\frac{1}{4} \tilde{h}^{\alpha \alpha^{\prime}} \tilde{h}^{\beta \beta^{\prime}} k_{i j} \\
& \cdot\left[\frac{1}{R} C_{\alpha \beta}^{\gamma} \widetilde{\phi}_{\gamma}^{i}+\frac{1}{R} C_{\bar{\alpha} \beta}^{\gamma} \widetilde{\lambda}_{\underline{y}}^{i}+g_{R} C_{j k}^{i} \widetilde{\phi}_{\alpha}^{j} \widetilde{\phi}_{\beta}^{k}\right] \\
& \left.\cdot\left[\frac{1}{R} C_{\alpha^{\prime} \beta^{\prime}}^{\gamma^{\prime}} \tilde{\phi}_{\gamma^{\prime}}^{j}+\frac{1}{R} C_{\bar{\alpha}^{\prime} \beta^{\prime}}^{\gamma^{\prime}} \tilde{\lambda}_{\underline{\gamma}^{\prime}}^{j}+g_{R} C_{k l}^{j} \widetilde{\phi}_{\alpha^{\prime}}^{k} \widetilde{\phi}_{\beta^{\prime}}^{l}\right]\right\} \sqrt{\gamma} d^{4} x,
\end{aligned}
$$

where

$$
\gamma=\left|\operatorname{det}\left(\gamma_{\mu \nu}\right)\right| \quad \text { and } \quad \tilde{\tau}_{S / I} \equiv \tau_{S / I}(\tilde{h})
$$

and where

$$
\begin{aligned}
D_{\mu} \tilde{h}_{\alpha \beta} & =\partial_{\mu} h_{\alpha \beta}+g_{S} C_{\alpha \hat{\gamma}}^{\delta} \tilde{A}_{\mu}^{\hat{\gamma}} \tilde{h}_{\delta \beta}+g_{R} C_{\hat{\gamma} \beta}^{\delta} \tilde{A}_{\mu}^{\hat{\gamma}} \tilde{h}_{\alpha \delta}, \\
D_{\mu} \widetilde{\phi}_{\alpha}^{i} & =\partial_{\mu} \widetilde{\phi}_{\alpha}^{i}+g_{S} C_{\alpha \hat{\gamma}}^{\delta} \tilde{A}_{\mu}^{\hat{\gamma}} \tilde{\phi}_{\delta}^{i}+g_{R} C_{\hat{i} j}^{i} \tilde{A}_{\mu}^{\hat{i}} \tilde{\phi}_{\alpha}^{j} .
\end{aligned}
$$

The previous action is still dimensionless but now, all quantities have standard dimensions: let $[L]$ be some length, then

$$
\begin{gathered}
\tau_{M} \sim L^{-2}, \quad \tilde{\tau}_{S / I} \sim L^{0}, \quad \tilde{A}_{\mu}^{\hat{\alpha}} \sim \tilde{A}_{\mu}^{\hat{i}} \sim L^{-1}, \quad \tilde{F}_{\mu \nu}^{\hat{\alpha}} \sim \tilde{F}_{\mu \nu}^{\hat{i}} \sim L^{-2}, \\
\tilde{h}_{\alpha \beta} \sim \tilde{h}^{\alpha \beta} \sim L^{0}, \quad \tilde{\phi}_{\alpha}^{i} \sim \tilde{\lambda}_{\gamma}^{i} \sim L^{-1}, \quad R^{2} \sim K \sim L^{2} .
\end{gathered}
$$

Notice that all coupling constants can be expressed in terms of the independent quantities $K, R$, and $g_{R} ; K$ is the Newton constant $K=16 \times 10^{-66} \mathrm{~cm}^{2}, R$ is a fundamental length (in $\mathrm{cm}$ ) and $g_{R}$ is a dimensionless constant; the relation $g_{s}^{2}=\frac{16 \pi K}{R^{2}}$ indicates that if $g_{s}^{2} \leqq 1$ then $R \geqq 10^{-33} \mathrm{~cm}$. If we expand the "internal" metric $\tilde{h}_{\alpha \beta}$ around some fixed background $\tilde{h}_{\alpha \beta}^{0}$, we obtain a constant $\frac{1}{16 \pi K R^{2}} \tilde{\tau}_{S / I}^{0}$ to be identified with the cosmological term $\frac{-2 \Lambda}{16 \pi K}$, that is $\Lambda=-\tau_{S / I}^{0} / 2 R^{2}$. Here, we do not get any contribution from the Higgs mechanism for $\phi_{\alpha}^{i}$ since the potential is zero at the minimum (see Sect. 5.3).

c) Normalization of Generators. Notice that in a conventional theory, one introduces usually different coupling constants for the simple components of the gauge group; also, one makes use of the Killing metric associated to each simple component. Finally the covariant derivatives, acting for example on the Higgs field which belongs to some representation $\varrho$ of the gauge group, are written by using a representation of the generators with some standard normalization. In our case, 
however, the simple components of $N(I) / I$ [or of $Z(\lambda(I))]$ are coupled to the Higgs fields via the same $g_{s}\left(\right.$ or $\left.g_{R}\right)$; also, the covariant derivative acting on the Higgs field is written by using the structure constants of $S-$ or $G-$ [see Eq. (5.4.1)]. In order to make contact with a conventional theory, one has therefore to specify the representation content for the Higgs fields and to rescale our generators (or the fields) according to some conventional normalization.

One obtains different coupling constants for the simple components of $Z(\lambda(I))$ [or of $N(I) / I]$ but their ratios (mixing angles) become entirely computable quantities.

\subsection{Model Building}

One can distinguish two classes of models: those where the group $N(I) / I$ is discrete and those where this is not the case; all models studied so far in the literature belong to the first category $[2,3,5,30]$. One has just to choose an extended spacetime $E$ which can be written locally as $M \times S / I$; when the pair $(S, I)$ (symmetric or not) is an isotropy irreducible space, the group $N(I) / I$ is indeed discrete and we are in the first situation; notice that Tables 1 and 2 of [1] can be helpful to provide examples where $N(I) / I$ is not discrete. In any case, this choice being made, one has to choose a group $R$ containing a subgroup isomorphic with $I$ (or $I$ divided by a normal subgroup of $I$ ), this allows one to define the group homomorphism $\lambda: I$ $\rightarrow \lambda(I) \subset R$. As already stressed, this map $\lambda$ does not characterize the "geometry" but rather the action of $S$ on the local product $E \times R$. If we now choose a $\operatorname{Lie}(R)$ valued, $S$ invariant Yang-Mills field on $E$ and a $S$ invariant metric on $E$, the dimensionally reduced Einstein-Yang-Mills field lagrangian will in particular contain a Lie $(Z(\lambda(I))+\operatorname{Lie}(N(I) / I)$ valued Yang-Mills field on $M$. In general one chooses the group $R$ big enough, in such a way that the centralizer $Z(\lambda(I))$ of $\lambda(I)$ is not discrete, but one could of course find an extreme situation where $Z(\lambda(I))$ is discrete and where $N(I) / I$ is the only piece left! (see example below). The Higgs field will now belong to some representation of the final gauge group $Z(\lambda(I)) \times N(I) / I$, and this representation can be found through the technique explained in Sect. 5.2. If one is now interested in possible "symmetry breaking," one should use the comments of Sect. 5.3; in particular, if one wants to be in a situation such that the potential $V(\phi)=0$, then, rather than choosing $\lambda$, one can directly construct a global homomorphism $\Lambda$ from $S$ into $R$ by choosing a group $R$ containing a subgroup isomorphic with $S$ (or divided by a normal subgroup). The final work is of course to restore the dimensionfull constants (using Sect. 5.4) and to analyze the physical spectrum of the model. Let us now analyze several examples:

1) $S=\mathrm{SO}(3)$ [respectively $S U(2)], I=\mathrm{SO}(2)=\mathrm{U}(1), R=\mathrm{SU}(3)$. These models have already been studied in [3]. $S / I$ is the two sphere $S^{2}$. The homomorphism $\lambda$ maps $I=\mathrm{SO}(2)=\mathrm{U}(1)$ onto a $\mathrm{U}(1)$ subgroup of $\mathrm{SU}(3)$. From the one hand, the centralizer of the image is $Z=\mathrm{SU}(2) \times \mathrm{U}(1) / Z_{2}$, from the other hand, the normalizer $N(I)$ of $\mathrm{SO}(2)$ in $\mathrm{SO}(3)$ [respectively $\mathrm{SU}(2)$ ] is $\mathrm{SO}(2) \times Z_{2}$ [respectively $\mathrm{SO}(2)]$, therefore $N(I) / I=Z_{2}$ (respectively $[e]$ ) is discrete. The Lie algebra of the final gauge group emerging from the reduction of the Einstein-Yang-Mills system is $\operatorname{Lie}(\mathrm{SU}(2) \times \mathrm{U}(1))$; in this case the Einstein part of the Lagrangian on $M \times S^{2}$ does not bring anything new (but for a factor $Z_{2}$ ); also the $h_{\alpha \beta}$ field is quite trivial since $S^{2}$ admits only one - up to scale - SO(3) [respectively SU(2)] 
invariant metric: $h_{\alpha \beta}(x)$ is therefore a real valued function $h(x)$ and is even a constant if we keep fixed the volume of $S / I$; then $\mathrm{KE}(h)=0$ and $V(h)$ is just a (cosmological) constant. The branching rules for the adjoint representation of $\mathrm{SO}(3)$ or $\mathrm{SU}(2)$ into $\mathrm{U}(1)$ and of the adjoint of $\mathrm{SU}(3)$ into $\mathrm{SU}(2)$ are

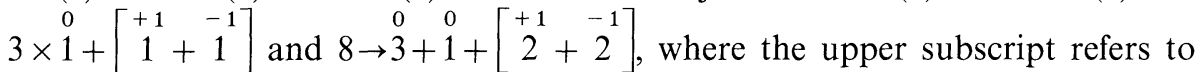
the U(1) eigenvalues; these eigenvalues can be obtained by writing $3 \times 3=8+1$ and by specifying the eigenvalues of the $U(1)$ generator (hypercharge) in the fundamental representation (3) of $\mathrm{SU}(3)$, a conventional choice is $\operatorname{diag}(2 / 3 ;-1 / 3,-1 / 3)$. The most general $\phi_{\alpha}^{i}$ field would map the $\left[\begin{array}{cc}+1 & -1 \\ 1 & +1\end{array}\right]$ subspace of $\operatorname{Lie}(\mathrm{SU}(2))$ into the $\left[\begin{array}{cc}+1 \\ 2+2\end{array}\right]$ subspace of $\operatorname{Lie}(\operatorname{SU}(3))$; however we can further specify the model by considering only those $\phi$ fields which map 1 into 2 and whose restriction to 1 is just zero. This last choice allows us to make contact with the phenomenology of the Weinberg Salam model where $\phi$ is a doublet of SU(2) with hypercharge +1 . Notice that here there is no direct coupling of $\phi$ to the field strength [Eq. (4.4.5)], $\phi_{\hat{\alpha}}^{\hat{i}}$ is zero since $N(I) / I$ is discrete. Absolute minima $V(\phi)=0$ for the Higgs potential are associated with the existence of algebra homomorphism $\Lambda$ from $\operatorname{Lie}(S)$ into $\operatorname{Lie}(R)$ - see Sect. 5.3. The group $\mathrm{SU}(3)$ has two maximal subgroups (defined up to conjugacy) $=\frac{\mathrm{SU}(2) \times \mathrm{U}(1)}{Z_{2}}$ and $\mathrm{SO}(3)$; correspondingly, the Lie algebra of $\mathrm{SU}(3)$ has two maximal simple subalgebras that we call $\mathrm{Lie}(\mathrm{SU}(2))$ and $\mathrm{Lie}(\mathrm{SO}(3))$ although they are isomorphic. The homomorphism $\lambda$ from $I=\mathrm{U}(1) \subset S=\mathrm{SU}(2)$ into Lie(SU(3)) can be extended in two possible ways: either we set $\Lambda(\operatorname{Lie}(S))$ $=\mathrm{Lie}(\mathrm{SU}(2))$ or we set $\Lambda(\mathrm{Lie}(S))=\mathrm{Lie}(\mathrm{SO}(3))$. Only in the first case the stabilizer of the minimum (the "unbroken" gauge group), i.e. the centralizer of $\Lambda(\mathrm{Lie}(\mathrm{SU}(2)))$ in $\mathrm{Lie}(\mathrm{SU}(3))$, is not zero, we get $\mathrm{Lie}(\mathrm{U}(1))$; indeed the centralizer of $\mathrm{SU}(2)$ in $\mathrm{SU}(3)$ is $U(1)$ whereas the centralizer of $\mathrm{SO}(3)$ in $\mathrm{SU}(3)$ is discrete $[(\mathrm{SU}(3) / \mathrm{SO}(3)$ is an irreducible symmetric space]. Notice that there is a difference between the two cases because an algebra homomorphism cannot necessarily be lifted to a group homomorphism: there are homomorphisms $\mathrm{SO}(3) \rightarrow \mathrm{SO}(3), \mathrm{SU}(2) \rightarrow \mathrm{SU}(2)$, and $\mathrm{SU}(2) \rightarrow \mathrm{SO}(3)$ but no homomorphism $\mathrm{SO}$ (3) $\rightarrow \mathrm{SU}(2)$. One can now compute mixing angles [3].

2) $S=\mathrm{U}(2, H)=\mathrm{Sp}(2)-2$ by 2 unitary matrices over the quaternions $H$. $I=\mathrm{U}(1, H)=\mathrm{Sp}(1)=\mathrm{SU}(2) ; R=\mathrm{SU}(5)$. The "internal" space $S / I$ is the seven sphere $S^{7}$. The maximal subgroup of $\mathrm{SU}(5)$ are $\mathrm{SU}(4) \times \mathrm{U}(1), \mathrm{SU}(3) \times \mathrm{SU}(2)$ $\times \mathrm{U}(1)$ and $\mathrm{Sp}(2)$ (in this section we no longer mention the discrete $Z_{p}$ factors). We choose $\lambda$ as a homomorphism from SU(2) onto an SU(2) subgroup of SU(5). From the one hand, the centralizer of the image is $S U(3) \times U(1)$, from the other hand, the normalizer $N(I)$ of $I=\mathrm{Sp}(1)$ in $\mathrm{Sp}(2)$ is $\mathrm{Sp}(1) \times \mathrm{Sp}(1)=\mathrm{SU}(2) \times \mathrm{SU}(2)$, as is clear by representing $\mathrm{Sp}(2)$ by $2 \times 2$ matrices over the quaternions; therefore we get in this case a non-discrete Lie group $N(I) / I=\mathrm{SU}(2)$. The full gauge group emerging from the reduction of the Einstein-Yang-Mills system is therefore $S U(3) \times S U(2) \times U(1)$. Besides the SU(2), the Einstein part of the lagrangian on $M \times S^{7}$ brings us also a non-trivial $h_{\alpha \beta}$ field; indeed, the space of $\operatorname{Sp}(2)$ invariant metrics on $S^{7}$ has 
dimension $d=7$ (one decomposes the tangent space at the origin of $S^{7}$ into $\operatorname{Ad}(\mathrm{Sp}(1))$ real-irreducible representations: $7=(1+1+1)+4$ and construct an $\operatorname{Ad}(\operatorname{Sp}(1))$ invariant bilinear symmetric form, therefore $d=\frac{3 \times 4}{2}+1=7$, see also [1], Sect. 4.1).

Let us now consider the Higgs field $\phi$; the branching rule for the adjoint representation of $\mathrm{Sp}(2)$ into $\mathrm{Sp}(1)=\mathrm{SU}(2)$ and of $\mathrm{SU}(5)$ into $\mathrm{SU}(3) \times \mathrm{SU}(2)$ are $[10] \rightarrow[3]+[1+1+1]+[2+\overline{2}]$ and $24 \rightarrow(8,1)+(1,3)+(3, \overline{2})+(\overline{3}, 2)+(1,1)$ where the upper subscript refers to the eigenvalue of the U(1) generator $Y$. These eigenvalues are obtained by specifying (arbitrarily) $Y=(2 / 3,2 / 3,2 / 3,-1,-1)$ in the fundamental representation 5 of SU(5) - as in the conventional SU(5) model of Georgi - Glashow - and writing $5 \times 5=24+1$. The most general Higgs field $\phi_{\alpha}^{i}$ would map the $[1+1+1]+[2+\overline{2}]$ subspace of $\operatorname{Lie}(\operatorname{Sp}(2))$ into the $(3,2)+(\overline{3}, 2)$ $+(1,1)$ subspace of $\operatorname{Lie}(\mathrm{SU}(5))$; however we can further specify the model by imposing that the restriction of $\phi$ to $1+1+1+\overline{2}$ vanishes; in such a way we obtain an SU(3) triplet of Higgs with hypercharge 5/3. In this theory we obtain a Weinberg angle equal to the one found in the conventional SU(5) model but the analogy stops there: we can find a homomorphism $\Lambda$ of $S=\operatorname{Sp}(2)$ onto $\Lambda(S)$ $=\mathrm{Sp}(2) \subset \mathrm{SU}(4) \subset \mathrm{SU}(5)$, the centralizer of $\Lambda(S)$ in $\mathrm{SU}(5)$ is then $\mathrm{U}(1)$, the $\mathrm{SU}(3)$ group is "broken" and the $N(I) / I=\mathrm{SU}(2)$ piece of the gauge group stays unbroken; this example is therefore for illustration only but cannot be used in phenomenology. Notice finally that, with the above assignment for the Higgs field, there is no direct coupling between the Higgs field and $F_{\mu \nu}^{\hat{\alpha}}$ or $F_{\mu \nu}^{\hat{i}}$, this would not be true if we suppose that $\phi_{\hat{\alpha}}^{\hat{i}}$ is not zero.

3) Let us conclude this section by just giving an example where $N(I) / I$ is not discrete but where $Z(\lambda(I))$ is discrete: $S=\mathrm{SO}(5), I=\mathrm{SO}(3)$, and $R=\mathrm{SO}(4)$. $S / I=V_{5,2}$ is the Stiefel manifold of 2-planes in $R^{5}$ and $\frac{N(I)}{I}=\mathrm{SO}(2)=\mathrm{U}(1)$, but if $\lambda(I)=\mathrm{SO}(3) \subset \mathrm{SO}(4)$, then $Z(\lambda(I))$ is discrete. Notice that

$$
V_{5,2}=\frac{\mathrm{SO}(5)}{\mathrm{SO}(3)} \neq S^{7}=\frac{\mathrm{Sp}(2)}{\mathrm{Sp}(1)}
$$

although $\mathrm{SO}(5)$ - respectively $\mathrm{SO}(3)-$ is isomorphic with $\frac{\mathrm{Sp}(2)}{Z_{2}}-$ respectively
$\mathrm{Sp}(1)$ $\frac{\mathrm{Sp}(1)}{Z_{2}}$.

\section{References}

1. Coquereaux, R., Jadczyk, A.: Geometry of multidimensional universes. Commun. Math. Phys. 90, 79-100 (1983)

2. Chapline, G., Slanski, R.: Dimensional reduction and flavor chirality. Nucl. Phys. B209, 461-483 (1982)

3. Manton, N.S.: A new six dimensional approach to the Weinberg-Salam model. Nucl. Phys. B158, 141-153 (1979)

4. Chapline, G., Manton, N.S.: The geometrical significance of certain Higgs potentials. Nucl. Phys. B184, 391 (1981)

5. Fairlie, D.B.: The interpretation of Higgs fields as Yang-Mills fields. In: Lecture Notes in Physics, Vol. 129, pp. 45-50. Berlin, Heidelberg, New York: Springer 1980 
6. Mayer, M.E.: Geometric aspects of symmetry breaking in gauge theories. In: Lecture Notes in Physics, Vol. 116, pp. 291-295. Berlin, Heidelberg, New York: Springer 1980

7. Jackiw, R.: In: Schladming Lectures. Acta Physica Austriaca Suppl. XXII, pp. 383-438. Berlin, Heidelberg, New York: Springer 1980

8. Jadczyk, A., Pilch, K.: Geometry of gauge fields in a multidimensional universe. Lett. Math. Phys. 8, 97-104 (1984)

9. Hudson, L.B., Kantowski, R.: Higgs fields from symmetric connections, the bundle picture. Oklahoma preprint (1983)

10. Cho, Y.M.: Higher-dimensional unifications of gravitation and gauge theories. J. Math. Phys. 16, 2029 (1975)

11. Cho, Y.M., Freund, P.G.O.: Non-abelian gauge fields as Nambu Goldstone fields. Phys. Rev. D12, 1711 (1975)

12. Jensen, G.R.: Einstein metrics on principal fiber bundles. J. Differ. Geom. 8, 599 (1973)

13. Coquereaux, R.: Dimensional reduction, Kaluza-Klein, Einstein spaces and symmetry breaking. Szczyrk Lectures 1983. Acta Phys. Pol Vol. B 15, 821-846 (1984)

14. Jadczyk, A.: Symmetry of Einstein-Yang-Mills systems and dimensional reduction. Banach Center Lectures 1983. J. Geom. Phys. (to appear)

15. Trautman, A.: On groups of gauge transformations. In: Lecture Notes in Physics, Vol. 129. Berlin, Heidelberg, New York: Springer 1980

16. Cho, Y.M.: Gauge theories on homogeneous fiber bundle. CERN Preprint TH 3414 (1982)

17. Jadczyk, A.: In: Supersymmetry and supergravity 1983. Proceedings of the XIX Karpacz Winter School, Milewski, B., ed. Singapore: World Scientific, 1983

18. Bergmann, P., Flaherty, E.: Symmetries in gauge theories. J. Math. Phys. 19, 212 (1978)

19. Forgacs, P., Manton, N.S.: Space-time symmetries in gauge theories. Commun. Math. Phys. 72, 15-35 (1980)

20. Harnad, J., Schnider, S., Vinet, L.: Group actions on principal bundles and invariance conditions for gauge fields. J. Math. Phys. 21 (12) (1980)

21. Harnad, J., Shnider, S., Tafel, J.: Group action on principal bundles and dimensional reduction. Lett. Math. Phys. 4, 107-113 (1980)

22. Palais, S.R.: The classification of G-spaces. Memoirs of the AMS No. 36. Providence, RI: AMS 1960

23. Bredon, G.E.: Introduction to compact transformation groups. New York: Academic Press 1972

24. Kastler, D.: Lectures on differential geometry (Marseille) (unpublished)

25. Kobayashi, S., Nomizu, K.: Foundations of differential geometry, Vols. I and II. New York: Interscience 1963

26. Michel, L.: Symmetry defects and broken symmetry, configurations, hidden symmetry. Rev. Mod. Phys. 52, 617-651 (1980)

27. Combe, Ph., Sciarrino, A., Sorba, P.: On the directions of spontaneous symmetry breaking in SU $(n)$ gauge theories. Nucl. Phys. B158, 452-468 (1979)

28. Girardi, G., Sciarrino, A., Sorba, P.: Some relevant properties of $\mathrm{SO}(n)$ representations for grand unified theories. Nucl. Phys. B182, 477-504 (1981)

29. Nagano, T.: A problem on the existence of an Einstein metric. J. Math. Soc. Jpn. 19, 30 (1967)

30. Forgacs, P., Zoupanos, G.: Dimensional reduction and dynamical symmetry breaking. CERN preprint $3883(1984)$

Communicated by G. Mack

Received June 26, 1984 